\title{
Exact Augmented Perpetual Manifolds: Corollary about Different Mechanical Systems with Exactly the Same Motions
}

\author{
Fotios Georgiades \\ Center for Nonlinear Systems, Chennai Institute of Technology, Chennai, India \\ Correspondence should be addressed to Fotios Georgiades; dr.fotios.georgiadis.gr@gmail.com
}

Received 16 April 2021; Accepted 14 July 2021; Published 10 September 2021

Academic Editor: Julius Kaplunov

Copyright ( $\odot 2021$ Fotios Georgiades. This is an open access article distributed under the Creative Commons Attribution License, which permits unrestricted use, distribution, and reproduction in any medium, provided the original work is properly cited.

\begin{abstract}
Perpetual points have been defined in mathematics recently, and they arise by setting accelerations and jerks equal to zero for nonzero velocities. The significance of perpetual points for the dynamics of mechanical systems is ongoing research. In the linear natural, unforced mechanical systems, the perpetual points form the perpetual manifolds and are associated with rigid body motions. Extending the definition of perpetual manifolds, by considering equal accelerations, in a forced mechanical system, but not necessarily zero, the solutions define the augmented perpetual manifolds. If the displacements are equal and the velocities are equal, the state space defines the exact augmented perpetual manifolds obtained under the conditions of a theorem, and a characteristic differential equation defines the solution. As a continuation of the theorem herein, a corollary proved that different mechanical systems, in the exact augmented perpetual manifolds, have the same general solution, and, in case of the same initial conditions, they have the same motion. The characteristic differential equation leads to a solution defining the augmented perpetual submanifolds and the solution of several types of characteristic differential equations derived. The theory in a few mechanical systems with numerical simulations is verified, and they are in perfect agreement. The theory developed herein is supplementing the already-developed theory of augmented perpetual manifolds, which is of high significance in mathematics, mechanics, and mechanical engineering. In mathematics, the framework for specific solutions of many degrees of freedom nonautonomous systems is defined. In mechanics/physics, the wave-particle motions are of significance. In mechanical engineering, some mechanical system's rigid body motions without any oscillations are the ultimate ones.
\end{abstract}

\section{Introduction}

Perpetual points (PPs) have been defined in mathematics recently [1]. The PPs of a dynamical system are obtained by setting accelerations and jerks of the equations of motion of a mechanical system equal to zero for nonzero velocities. Currently, there are four research directions relevant to the perpetual points. The first research direction, including experiments, is strictly relevant to developing the perpetual point theory [1-4]. The second research direction is to identify hidden and chaotic attractors [5-13]. The third research direction is to identify dissipative systems [14-18], and the fourth direction is about their use in mechanics [19-22]. In [19, 20] with proved theorems, the PPs in linear unforced natural mechanical systems are associated with rigid body modes/motions, and they form the perpetual manifolds.
Moreover, the perpetual manifolds of some nonlinear unforced systems which are associated with rigid body motions in $[19,20]$ are shown. Based on the correlation of the PPs with the rigid body modes/motions, some new definitions of mechanical systems in [22] are derived. The mechanical systems that admit rigid body modes as solutions are called perpetual mechanical systems [22]. The perpetual manifold's definition extended in [22] to the augmented perpetual manifolds defined by the state space of the solutions of external forced mechanical systems motions that all the accelerations are equal but not necessarily zero. The state spaces, with the solutions that all generalized coordinates are equal and the velocities are equal, are called exact augmented perpetual manifolds. Based on these new definitions, a theorem written in [21] and proved in [22] defines the conditions for exact augmented perpetual manifolds solutions of mechanical systems. A corollary 
proved that, in exact augmented perpetual manifolds of harmonic excitation, the N-DOF mechanical system's motion is particle wave. The theorem is applied in three examples, where, in one of them, explicit solution of two types of external forces in the exact augmented perpetual manifolds is defined.

There are significant research efforts in examining rigid body equations' validity in modeling elastic structures, for example, $[23,24]$. The examination of a viscoelastic bar with end loads targeting application in rails, including more significant terms in modeling and further in the nonlinear dynamic analysis, is done in [23]. Also, in [24], the plane and antiplane dynamic problems of an elastic rectangle, with asymptotic and exact dynamic analysis, were examined to identify the limitations of the rigid body formalism in elastic structures.

This article continues the work done in [22] by proving a corollary arising from the theorem in [21, 22]. The explicit analytical form of the exact augmented perpetual manifolds for several external forces is then given.

In the numerical section, to validate the analytical results, three examples are examined.

The first example is a two-DOF mechanical system in translational motion. A shaft in torsional motion with a nonlinear energy sink attachment, examined through finite element model, is the second example. The third example is a five-degrees-of-freedom mechanical system.

\section{Theory}

In Section 2.1, the theory developed in [22], relevant to the corollary, is presented. In Section 2.2, there are the statement and the proof of corollary. The last section, Section 2.3, is about the augmented perpetual submanifold's explicit analytical forms for specific external forces.

2.1. Preliminary. In this section, preliminary definitions and theory, developed in other articles, are presented. The perpetual points have been defined recently as the sets of points that arise when the accelerations and jerks of the equations describing the motion of a mechanical system, for nonzero velocities, are set equal to zero [1].

In cases where the perpetual points are not just a few points but infinite, they form the perpetual manifolds [20]. A mechanical system that admits rigid body motions as a solution is called a perpetual mechanical system [22], and the rigid body motions with perpetual manifolds $[19,20]$ are associated. The concept of perpetual manifolds in [22] extended to augmented perpetual manifolds, which are defined when all mechanical systems' accelerations are equal to each other but not necessarily equal to zero [22]. The augmented perpetual manifolds of a mechanical system, where all the generalized velocities are equal and the generalized displacements are equal, form the exact augmented perpetual manifolds [22]. Based on the exact augmented perpetual manifolds definition, the following theorem stated in [21] and proved in [22] arose:

Any $N(\geq 2)$-degrees-of-freedom discrete mechanical system with generalized coordinates $q_{i}(t)$ can be written as a perpetual mechanical system with external forcing that is described by the following system of differential equations:

$$
\begin{aligned}
& {\left[\mathbf{M}_{\mathbf{i}, \mathbf{j}}\left(t, q_{l}(t), \dot{q}_{m}(t)\right)\right] \times\left\{\ddot{q}_{i}(t)\right\}+\left[\mathbf{C}_{\mathbf{i}, \mathbf{j}}\right] \times\left\{\dot{q}_{i}(t)\right\}+\left[\mathbf{K}_{\mathbf{i}, \mathbf{j}}\right] \times\left\{q_{i}(t)\right\}+\left\{F_{i}^{N L}\left(q_{n}(t), \dot{q}_{o}(t)\right)\right\}} \\
& =\left\{F_{i}\left(t, q_{p}(t), \dot{q}_{q}(t)\right)\right\} \quad \text { for } i=1, \ldots, N, j=1, \ldots, N, l, m, n, o, p, q \in\{1,2, \ldots, N\}\left(q_{i}(t), \dot{q}_{i}(t), \ddot{q}_{i}(t)\right) \in \mathbb{R}^{3},
\end{aligned}
$$

and it admits unique solutions for the following matrices: $\left[\mathbf{M}_{\mathbf{i}, \mathbf{j}}\right]$ is a real $N \times N$ inertia matrix with elements that can be nonsmooth, nonlinear, and time- and state-dependent functions but having at least one nonzero sum of $k$-row for all time instants, $\left[\mathbf{K}_{\mathbf{i}, \mathbf{j}}\right]$ and $\left[\mathbf{C}_{\mathbf{i}, \mathbf{j}}\right]$, are real $N \times N$ constant, stiffness and proportional to velocity vector, matrices, $\left\{F_{i}^{\mathrm{NL}}\right\}$ is an $N \times 1$ vector of nonlinear internal forces with elements that can be state-dependent nonlinear functions which can be nonsmooth but single-valued for rigid body motions, and $F_{i}^{\mathrm{NL}}\left(q_{s}, 0\right)=0$ for $q_{s} \in \mathbb{R},\left\{F_{i}\right\}$ is a real $N \times 1$ vector of external forces with elements that can be time- and statedependent and may be nonlinear and nonsmooth functions, if the external forces $\left(F_{i}\right)$ with the reference $k$-inertia external force $\left(F_{k}\right)$ are related as follows:

$$
F_{i}\left(t, q_{a}(t), \dot{q}_{a}(t)\right)=\frac{\sum_{j=1}^{N} M_{i, j}\left(t, q_{a}(t), \dot{q}_{a}(t)\right) \cdot F_{k}\left(t, q_{a}(t), \dot{q}_{a}(t)\right)}{\sum_{j=1}^{N} M_{k, j}\left(t, q_{a}(t), \dot{q}_{a}(t)\right)}, \quad \text { for } i, k \in\{1,2, \ldots, N\}, \text { and } q_{a}(t)=q_{i}(t), \dot{q}_{a}(t)=\dot{q}_{i}(t),
$$


and then the solution of any of the following differential equations,

$$
\ddot{q}_{a}(t)=\frac{F_{k}\left(t, q_{a}(t), \dot{q}_{a}(t)\right)}{\sum_{j=1}^{N} M_{k, j}\left(t, q_{a}(t), \dot{q}_{a}(t)\right)}=G\left(t, q_{a}(t), \dot{q}_{a}(t)\right),
$$

with vector field $G$, for the following set of initial conditions at the time instant $t_{0}$,

$$
\begin{aligned}
& q_{i}\left(t_{0}\right)=q_{a}\left(t_{0}\right), \quad \text { for } i=1, \ldots, N, \text { and } q_{a}\left(t_{0}\right) \in \mathbb{R}, \\
& \dot{q}_{i}\left(t_{0}\right)=\dot{q}_{a}\left(t_{0}\right), \quad \text { for } i=1, \ldots, N \text { and } q_{a}\left(t_{0}\right) \in \mathbb{R},
\end{aligned}
$$

is defining the generalized coordinates $q_{i}$ and their velocities in the exact augmented perpetual manifold, $\mathbf{X}_{\mathbf{a}}=$ $\left\{\left(t, q_{a}(t), \ldots, q_{a}(t), \dot{q}_{a}(t), \ldots, \dot{q}_{a}(t)\right),\left(t, q_{a}(t), \dot{q}_{a}(t)\right) \in \mathbb{R}^{3}\right\}$ (see (5)) $[21,22]$.

The basis of the exact augmented perpetual manifold is the augmented perpetual submanifold $\mathbf{A}$, and the following triple defines it [22]:

$$
\mathbf{A}=\left\{\left(t, q_{a}(t), \dot{q}_{a}(t)\right) \in \mathbb{R}^{3}\right\}
$$

In the next section, based on the theorem above, there is the corollary.

\subsection{Corollary}

Corollary 1. Two externally forced different discrete perpetual mechanical systems, with an exact augmented perpetual manifold which arises by the same vector field, have the same general solution in the exact augmented perpetual manifold. If they also have the same initial conditions, then the functions defining their motions are coinciding.

Proof. The exact augmented perpetual manifold solution by equation (3) arises with a vector field $G\left(t, q_{a}(t), \dot{q}_{a}(t)\right)$. Therefore, the two systems with the same vector field in equation (3) have the same general solution, and if they start their motion from the same initial conditions, they have the same functions defining their motion.

Therefore, equation (3) defines the exact augmented perpetual manifold as the characteristic differential equation of the exact augmented perpetual manifold and applies to any perpetual mechanical system. Unless otherwise stated, all perpetual mechanical systems, following the theorem requirements ending up to a solvable characteristic differential equation (3), have the same general solution.

In this corollary, the equations might be the same for angular and translational generalized coordinates, meaning that the two systems' inertia elements do not have the same motion, but the same functions define their motion.

2.3. Several Types of Exact Augmented Perpetual Manifolds. In this section, several types of external forces that fulfil the theorem's requirements are considered. Each system's motion is in an exact augmented perpetual manifold, described by equation (3) solutions.

In the exact augmented perpetual manifolds, the responses/velocities of all masses are the same, which means that the $N$-DOF system is moving like a particle with absolute synchronization of all displacements.

A time-dependent vector field in the exact augmented perpetual manifolds is given by

$$
\ddot{q}_{a}(t)=G\left(t, q_{a}(t), \dot{q}_{a}(t)\right)=\frac{F_{k}\left(t, q_{a}(t), \dot{q}_{a}(t)\right)}{\sum_{j=1}^{N} M_{k, j}\left(t, q_{a}(t), \dot{q}_{a}(t)\right)}=\frac{f_{k}(t)}{m}, \quad m \in \mathbb{R}_{\neq 0} .
$$

In case of constant inertia matrix, equation (6) takes the following form:

$$
\ddot{q}_{a}(t)=\frac{F_{k}\left(t, q_{a}(t), \dot{q}_{a}(t)\right)}{\sum_{j=1}^{N} M_{k, j}}=\frac{f_{k}\left(t, q_{a}(t), \dot{q}_{a}(t)\right)}{m}=G\left(t, q_{a}(t), \dot{q}_{a}(t)\right),
$$

and the denominator in the right-hand side is nonzero and positive for natural mechanical systems.
In case of a state-dependent inertia matrix, equation (6) for specific types of external forces takes the following form:

$$
\begin{aligned}
\ddot{q}_{a}(t) & =\frac{F_{k}\left(t, q_{a}(t), \dot{q}_{a}(t)\right)}{\sum_{j=1}^{N} M_{k, j}\left(t, q_{a}(t), \dot{q}_{a}(t)\right)}=\frac{\sigma \cdot \sum_{j=1}^{N} M_{k, j}\left(t, q_{a}(t), \dot{q}_{a}(t)\right) \cdot f_{k}\left(t, q_{a}(t), \dot{q}_{a}(t)\right)}{\sum_{j=1}^{N} M_{k, j}\left(t, q_{a}(t), \dot{q}_{a}(t)\right)}= \\
& =\sigma \cdot f_{k}\left(t, q_{a}(t), \dot{q}_{a}(t)\right)=\frac{f_{k}\left(t, q_{a}(t), \dot{q}_{a}(t)\right)}{m}=G\left(t, q_{a}(t), \dot{q}_{a}(t)\right) .
\end{aligned}
$$


and they are applied in $k^{\text {th }}$-mass. The theorem's validity requires that the rest of applied forces in all the other masses should be correlated through equation (2). In the case of a vector field given by the right-hand side of equation (6), equation (2) that defines the rest of forces takes the following form:

$$
\begin{aligned}
F_{i}\left(t, q_{a}(t), \dot{q}_{a}(t)\right) & =\frac{\sum_{j=1}^{N} M_{i, j}\left(t, q_{a}(t), \dot{q}_{a}(t)\right)}{\sum_{j=1}^{N} M_{k, j}\left(t, q_{a}(t), \dot{q}_{a}(t)\right)} \cdot F_{k}\left(t, q_{a}(t), \dot{q}_{a}(t)\right)= \\
& =\sum_{j=1}^{N} M_{i, j}\left(t, q_{a}(t), \dot{q}_{a}(t)\right) \cdot \sigma \cdot f_{k}\left(t, q_{a}(t), \dot{q}_{a}(t)\right), \quad \text { for }=i, k \in\{1,2, \ldots, N\} .
\end{aligned}
$$

Six types of external forces are considered, and, in Table 1 , the explicit forms of the displacements and velocities, for each considered type, in the exact augmented perpetual manifolds are provided. Irrespective of their original system equations of motion, these functions are the same for all mechanical systems with the same external forces in the exact augmented perpetual manifolds.

More precisely, each type of force $f_{k}^{(r)}$ leads to a solution $q_{a, r}(t)$ through equation (11) or (24) and the triple $\left(t, q_{a, r}, \dot{q}_{a, r}\right)$ forms the augmented submanifold $A^{(r)}$. Depending on the number of degrees of freedom, using the augmented submanifolds $\mathbf{A}^{(\mathbf{r})}$ as a basis, the exact augmented manifold $\mathbf{X}$ is formed.
The considered six external forces of Table 1 lead easily to an explicit solution of equation (6) as follows:

(1) Linear time varying forces $\left(f_{k}^{(1)}(t)\right)$ :

$$
\ddot{q}_{a, 1}(t)=\frac{f_{k}^{(1)}(t)}{m}=\frac{\eta \cdot t+c}{m}=G^{(1)}(t) \text {, with }(\eta, c) \in \mathbb{R}^{2} \text {. }
$$

The solution of equation (10) is defined in [22] and is shown in Table 1. This solution leads to the augmented perpetual submanifold $\mathbf{A}^{(1)}$ and is given by equations (a)-(f) in Table 1.

(2) Single frequency harmonic forces $\left(f_{k}^{(2)}\right)$ :

$$
\ddot{q}_{a, 2}(t)=\frac{f_{k}^{(2)}(t)}{m}=\frac{A_{\mathrm{ex}} \cdot \sin \left(\omega_{\mathrm{ex}} \cdot t+\theta_{\mathrm{ex}}\right)}{m}=G^{(2)}(t) \text {, with }\left(A_{\mathrm{ex}}, \theta_{\mathrm{ex}}\right) \in \mathbb{R}^{2} \text { and } \omega_{\mathrm{ex}} \in \mathbb{R}_{\geq 0} \text {, }
$$

where $A_{\mathrm{ex}}$ is the amplitude, $\omega_{\mathrm{ex}}$ and $\theta_{\mathrm{ex}}$ are the external frequency and phase, respectively, and the other forces should be in the form defined by equation (9). The solution in [22] is given and in Table 1 is presented. The augmented perpetual submanifold $\mathbf{A}^{(2)}$ is defined through the general solution given by equations (17b)-(18b) in Table 1 .

(3) Harmonic forces with $N_{f}$ - frequencies $\left(N_{f} \in \mathbb{N}^{*}\right)$, $\left(f_{k}^{(3)}(t)\right)$ :

$$
G^{(3)}(t)=\frac{f_{k}^{(3)}(t)}{m}=\frac{\sum_{i=1}^{N_{f}} A_{\mathrm{ex}, i} \cdot \sin \left(\omega_{\mathrm{ex}, i} \cdot t+\theta_{\mathrm{ex}, i}\right)}{m} \text {, with }\left(A_{\mathrm{ex}, i}, \theta_{\mathrm{ex}, i}\right) \in \mathbb{R}^{2 \cdot N_{f}} \text { and, } \omega_{\mathrm{ex}, i} \in \mathbb{R}_{\geq 0}^{N_{f}} \text {, }
$$

considering that the other forces are following equation (6), in the exact augmented perpetual manifolds, the motion is described by

$\ddot{q}_{a, 3}(t)=\frac{\sum_{i=1}^{N_{f}} A_{\mathrm{ex}, i} \cdot \sin \left(\omega_{\mathrm{ex}, i} \cdot t+\theta_{\mathrm{ex}, i}\right)}{m}=G^{(3)}(t)$

and $\dot{q}_{a, 3}(t)$ and $q_{a, 3}(t)$ are given similarly with single and double integrations of equation (12b), in time, respectively. The general solution of equation $(12 \mathrm{~b})$ is shown in Table 1 . The equations ((c)-(h) presented in
Table 1) can be used to define the augmented perpetual submanifold $\mathbf{A}_{\mathbf{N}_{\mathrm{f}}}^{(3)}$.

(4) Harmonic forces with $N_{f}$ - frequencies combined with a function $\ddot{V}(t)$ : for example, $\dot{V}(t)$ is monotonic for the considered time interval of motion, and the form of the force $\left(f_{k}^{(4)}(t)\right)$ is given by

$$
G^{(4)}(t)=\frac{f_{k}^{(4)}(t)}{m}=\frac{\sum_{i=1}^{N_{f}} A_{\mathrm{ex}, i} \cdot \sin \left(\omega_{\mathrm{ex}, i} \cdot t+\theta_{\mathrm{ex}, i}\right)+\ddot{V}(t)}{m},
$$

in case that the other forces follow equation (9), the motion is described by 


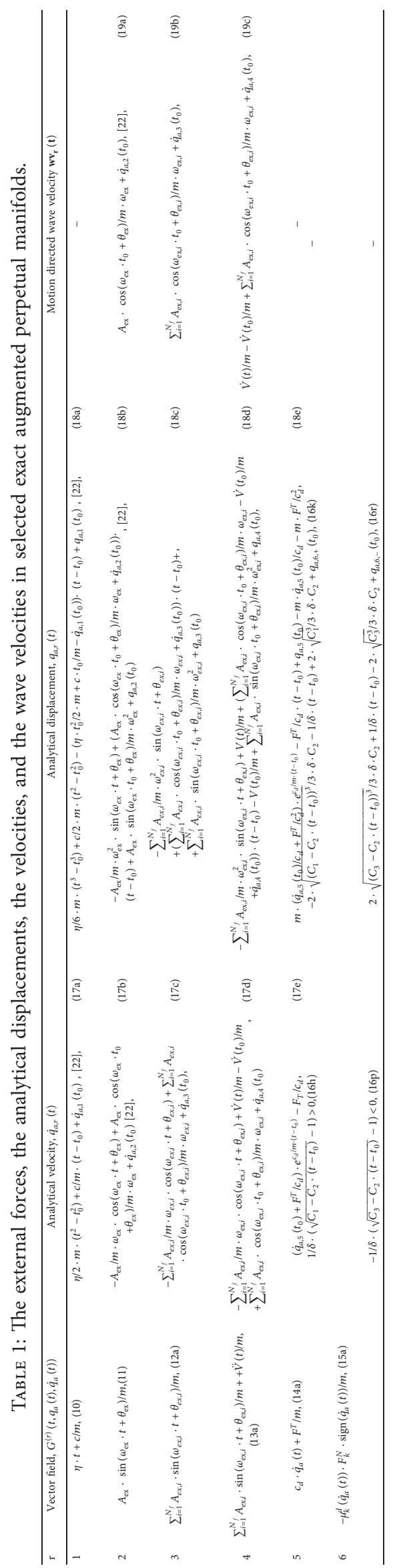


$q_{a, 4}(t)=\frac{\sum_{i=1}^{N_{f}} A_{\mathrm{ex}, i}, \sin \left(\omega_{\mathrm{ex}, i} \cdot t+\theta_{\mathrm{ex}, i}\right)+\ddot{V}(t)}{m}=G^{(4)}(t)$,

and, similarly, $\dot{q}_{a, 4}(t)$ and $q_{a, 4}(t)$, with direct integration in time of equation (13b), are given. The augmented perpetual submanifold $\mathbf{A}_{\mathbf{N}_{\mathrm{f}}, \ddot{\mathbf{V}}}^{(3)}$ is defined through equations (d)-(i) in Table 1.

(5) Aerodynamic and time-dependent force $\left(f_{k}^{(5)}\left(t, \dot{q}_{k, 5}(t)\right)\right)$ :

$$
f_{k}^{(5)}(t)=c_{d} \cdot \dot{q}_{k, 5}(t)+F^{T}(t), \text { with }\left(c_{d}, F^{T}\right) \in \mathbb{R}^{2} \text {. }
$$

For negative $c_{d}\left(c_{d} \in \mathbb{R}_{<0}\right)$, the force is aerodynamic drag, and, in case of positive $c_{d}\left(c_{d} \in \mathbb{R}_{>0}\right)$, it is a flutter. For positive $\dot{q}_{k, 5}(t)$, positive $F_{T}$ is a throttle or negative $F_{T}$ is a break and vice versa for negative $\dot{q}_{k, 5}(t)$. The other forces for the validity of theorem in the exact augmented perpetual manifold must follow equation (9) and must be given by

$$
f_{i}^{(5)}\left(t, \dot{q}_{a, 5}(t)\right)=\frac{\sum_{j=1}^{N} M_{i, j}\left(t, q_{a}(t), \dot{q}_{a}(t)\right)}{m} \cdot\left(c_{d} \cdot \dot{q}_{a, 5}(t)+F^{T}(t)\right) .
$$

Then, in the exact augmented perpetual manifolds, the solution is provided through equation (6) which takes the following form:

$\ddot{q}_{a, 5}(t)=\frac{c_{d} \cdot \dot{q}_{a, 5}(t)+F^{T}(t)}{m}=G^{(5)}\left(t, \dot{q}_{a}(t)\right)$.

For $F^{T}=c t\left(F_{T} \in \mathbb{R}_{\neq 0}\right)$, the following change of variables,

$z(t)=\dot{q}_{a, 5}(t)+\frac{F^{T}}{c_{d}} \Rightarrow \ddot{q}_{a, 5}=\dot{z}(t)=\frac{c_{d}}{m} \cdot z(t)$,

leads to the general solution of equations (14c) and (14d):

$$
z(t)=z\left(t_{0}\right) \cdot e^{c_{d} / m \cdot\left(t-t_{0}\right)}
$$

Once the velocities $\dot{q}_{a, 5}(t)$, using equation (14d), are defined, the displacements $q_{a, 5}(t)$, with direct integration of equation (14e) in time, can be obtained, and their forms are in Table 1. Equations (e) and (j) in Table 1 are used to define the augmented perpetual submanifold $\mathbf{A}^{(5)}$.
(6) A special type of considered external force $\left(f_{k}^{(6)}\right)$ is dry friction nonsmooth force in the following form [25]:

$$
f_{k}^{(6)}\left(t, \dot{q}_{k, 6}(t)\right)=-\mu_{k}^{d}\left(\dot{q}_{k, 6}(t)\right) \cdot F_{k}^{N} \cdot \operatorname{sign}\left(\dot{q}_{k, 6}(t)\right),
$$

where $F_{k}^{N} \in \mathbb{R}_{>0}$, is the normal force to the motion and $\mu_{k}^{d}$ is the dynamic friction coefficient which is dependent on velocity and is defined as in [25]:

$$
\mu_{k}^{d}\left(\dot{q}_{k, 6}(t)\right)=\frac{\mu_{k}^{s}}{1+\delta \cdot\left|\dot{q}_{k, 6}(t)\right|} \text {, with }\left(\mu_{k}^{s}, \delta\right) \in \mathbb{R}_{>0}^{2} .
$$

In the exact augmented perpetual manifold, equation (15a), considering equation (15b), takes the following form:

$$
f_{k}^{(6)}\left(t, \dot{q}_{a, 6}(t)\right)=-\left(\frac{\mu_{k}^{s}}{1+\delta \cdot\left|\dot{q}_{a, 6}(t)\right|}\right) \cdot F_{k}^{N} \cdot \operatorname{sign}\left(\dot{q}_{a, 6}(t)\right) .
$$

The other forces $\left(f_{i}^{(6)}\right)$ in the exact augmented perpetual manifolds must satisfy equation (6) and must be in the following form:

$$
f_{i}^{(6)}\left(t, \dot{q}_{a, 6}(t)\right)=\frac{\sum_{j=1}^{N} M_{i, j}\left(t, q_{a}(t), \dot{q}_{a}(t)\right)}{m} \cdot\left(-\left(\frac{\mu_{k}^{s}}{1+\delta \cdot\left|\dot{q}_{a, 6}(t)\right|}\right) \cdot F_{k}^{N} \cdot \operatorname{sign}\left(\dot{q}_{a, 6}(t)\right)\right) .
$$

The existence of the system's solutions is ensured when convexification of the external forces (equations (15c) and (15d)) takes place in all the equations, and then the system will be a Fillipov system that has solutions $[25,26]$.
In this case, the dynamics are described by equation (6), which takes the following explicit form:

$$
\ddot{q}_{a, 6}(t)=\frac{1}{m} \cdot\left(-\left(\frac{\mu_{k}^{s}}{1+\delta \cdot\left|\dot{q}_{a, 6}(t)\right|}\right) \cdot F_{k}^{N} \cdot \operatorname{sign}\left(\dot{q}_{a, 6}(t)\right)\right)=G^{(6)}\left(t, \dot{q}_{a}(t)\right) .
$$


A change of variables,

$$
w(t)=\dot{q}_{a, 6}(t),
$$

leads to

$$
\dot{w}(t)=-\frac{1}{m} \cdot\left(\frac{\mu_{k}^{s}}{1+\delta \cdot|w(t)|}\right) \cdot F_{k}^{N} \cdot \operatorname{sign}(w(t)),
$$

and, after convexification of the vector field, equation (16b) takes the following form:

$$
\dot{w}(t) \in\left\{\begin{array}{l}
-\frac{1}{m} \cdot\left(\frac{\mu_{k}^{s}}{1+\delta \cdot w(t)}\right) \cdot F_{k}^{N}, \quad w(t)>0, \\
\frac{1}{m} \cdot \mu_{k}^{s} \cdot F_{k}^{N} \cdot[-1,1], \quad w(t)=0, \\
\frac{1}{m} \cdot\left(\frac{\mu_{k}^{s}}{1-\delta \cdot w(t)}\right) \cdot F_{k}^{N}, \quad w(t)<0 .
\end{array}\right.
$$

Equation (16c) with differential inclusions forms a Fillipov system, and the solution arises by considering initial conditions in the three individual vector fields as follows.

In case of positive initial velocities in the mechanical system $\left(w\left(t_{0}\right) \in \mathbb{R}_{>0}\right)$, as long as the velocity is positive, the motion is described by the first vector field of equation (16c) and in explicit form is given by

$$
\dot{w}_{+}(t)=-\frac{1}{m} \cdot\left(\frac{\mu_{k}^{s}}{1+\delta \cdot w_{+}(t)}\right) \cdot F_{k}^{N} .
$$

Consider a change of variables,

$$
v(t)=1+\delta \cdot w_{+}(t),
$$

where $v(t) \neq 0$, and then equation (16d) takes the following form:

$$
\dot{v}(t)=-\frac{\delta \cdot \mu_{k}^{s} \cdot F_{k}^{N}}{m} \cdot \frac{1}{v(t)},
$$

which can be solved with integration and leads to

$$
v(t)=\sqrt{v^{2}\left(t_{0}\right)-\frac{2 \cdot \delta \cdot \mu_{k}^{s} \cdot F_{k}^{N}}{m} \cdot\left(t-t_{0}\right)},
$$

or taking into account equations (16a) and (16e) in equation (16g) leads to

$$
\dot{q}_{a, 6,+}(t)=\frac{1}{\delta} \cdot\left(\sqrt{C_{1}-C_{2} \cdot\left(t-t_{0}\right)}-1\right)>0, \quad \text { for } \dot{q}_{a, 6,+}(t) \in \mathbb{R}_{>0} \text {, }
$$

where

$$
\begin{aligned}
& C_{1}=\left(1+\delta \cdot \dot{q}_{a, 6}\left(t_{0}\right)\right)^{2} \text {, therefore } C_{1} \in \mathbb{R}_{>0}, \\
& C_{2}=\frac{2 \cdot \delta \cdot \mu_{k}^{s} \cdot F_{k}^{N}}{m}, \text { therefore } C_{2} \in \mathbb{R}_{>0},
\end{aligned}
$$

since all the involved parameters in equation (16j) are positive.

The displacements can be obtained with direct integration of equation (16h), and they are given by

$$
q_{a, 6,+}(t)=-\frac{2 \cdot \sqrt{\left(C_{1}-C_{2} \cdot\left(t-t_{0}\right)\right)^{3}}}{3 \cdot \delta \cdot C_{2}}-\frac{1}{\delta} \cdot\left(t-t_{0}\right)+\frac{2 \cdot \sqrt{C_{1}^{3}}}{3 \cdot \delta \cdot C_{2}}+q_{a, 6,+}\left(t_{0}\right) .
$$

In case that the initial velocities of the mechanical system are negative $\left(w\left(t_{0}\right) \in \mathbb{R}_{<0}\right)$, as long as the velocity is negative, the motion is described by the third vector field of equation (16c) and in explicit form is given by

$$
\dot{w}_{-}(t)=\frac{1}{m} \cdot\left(\frac{\mu_{k}^{s}}{1-\delta \cdot w_{-}(t)}\right) \cdot F_{k}^{N},
$$

and then consider the following change of variables:

$$
u(t)=1-\delta \cdot w_{-}(t),
$$

where $u(t) \neq 0$, and then equation (161) takes the following form:

$$
\dot{u}(t)=-\frac{\delta \cdot F_{k}^{N} \cdot \mu_{k}^{s}}{m} \cdot \frac{1}{u(t)},
$$

which after integration leads to

$$
u(t)=\sqrt{u^{2}\left(t_{0}\right)-\frac{2 \cdot \delta \cdot F_{k}^{N} \cdot \mu_{k}^{s}}{m} \cdot\left(t-t_{0}\right)},
$$

or taking into account equations $(16 \mathrm{a})$ and $(16 \mathrm{~m})$ in equation (16o) leads to

$$
\dot{q}_{a, 6,-}(t)=-\frac{1}{\delta} \cdot\left(\sqrt{C_{3}-C_{2} \cdot\left(t-t_{0}\right)}-1\right), \quad \text { for } \dot{q}_{a, 6,-}(t) \in \mathbb{R}_{<0},
$$

where

$$
C_{3}=\left(1-\delta \cdot \dot{q}_{a, 6,-}\left(t_{0}\right)\right)^{2}
$$

The displacements can be obtained with direct integration of equation (16p) and they are given by

$$
\begin{aligned}
q_{a, 6,-}(t)= & \frac{2 \cdot \sqrt{\left(C_{3}-C_{2} \cdot\left(t-t_{0}\right)\right)^{3}}}{3 \cdot \delta \cdot C_{2}}+\frac{1}{\delta} \cdot\left(t-t_{0}\right) \\
& -\frac{2 \cdot \sqrt{C_{3}^{3}}}{3 \cdot \delta \cdot C_{2}}+q_{a, 6,-}\left(t_{0}\right) .
\end{aligned}
$$


In any of the two described ways, the velocities tend to zero. Therefore, the three ways where the velocities are becoming zero are as follows:

(i) Through equation (16h)

(ii) Through equation (16p),

(iii) Through initial zero velocities, and then the system is remaining with zero velocity, and all the masses are in stick condition.

The exact augmented perpetual submanifold $\mathbf{A}^{(6)}$ is defined by the general solution of equations (16d) and (16l) which is given by equations (16h), (16k), (16p), and (16r).

The selected types of forces are some representative types of external forces in the perpetual mechanical system, which, of course, by no means are the types of the external forcing limited.

The external forces can be any combination of them, or any other type and nature, that fulfil equations (2) and (9). They can also be thermomechanical, electromechanical, or aerodynamic forces, and so forth. In such cases, the perpetual mechanical system's state space forms an exact augmented perpetual manifold, and the motion in the case of constant inertia matrices is described by the solution of the differential equations (3) and (6).

\section{Numerical Results}

The theory developed in the previous section in three examples of mechanical systems is applied. In the first example of a 2-degrees-of-freedom (DOF) system, the four external forces (3-6 of Table 1), with solutions determined in this article, are applied. In the second example of a shaft in torsional vibration with torsional nonlinear energy sink, modeled with finite element structural matrices, the first two types of forces with the analytical solution of [22] are applied, for the theory validation in many-degrees-of-freedom system. Finally, in the third example, a 5-DOF mechanical system, the first two types of external forces of the 2-DOF mechanical system are applied for comparison with the previous section's results.

3.1. A Two-Degrees-of-Freedom System. In this section, an example of a 2-DOF mechanical system is examined. Figure 1 shows the 2-DOF mechanical system in translational motion, and the equations of motion have the following form:

$$
\begin{gathered}
{\left[\begin{array}{cc}
m_{x, 0}+\dot{x}^{2} & 0 \\
0 & m_{y, 0}+\dot{y}^{2}
\end{array}\right] \times\{\ddot{x} \ddot{y}\}+\left[\begin{array}{cc}
k_{1} & -k_{1} \\
-k_{1} & k_{1}
\end{array}\right] \times\left\{\begin{array}{l}
x \\
y
\end{array}\right\}+\left[\begin{array}{cc}
c_{1} & -c_{1} \\
-c_{1} & c_{1}
\end{array}\right] \times\left\{\begin{array}{l}
\dot{x} \\
\dot{y}
\end{array}\right\}} \\
+\left\{\begin{array}{l}
k_{2} \cdot(x-y)^{3}+c_{2} \cdot \tanh (b \cdot(\dot{x}-\dot{y})) \\
k_{2} \cdot(y-x)^{3}+c_{2} \cdot \tanh (b \cdot(\dot{y}-\dot{x}))
\end{array}\right\}=\left\{\begin{array}{l}
F_{x}(t, x, y, \dot{x}, \dot{y}) \\
F_{y}(t, x, y, \dot{x}, \dot{y})
\end{array}\right\} .
\end{gathered}
$$

The system is comprised of two masses $\left(m_{\mathrm{x}}\right.$ and $\left.m_{\mathrm{y}}\right)$ that are velocity-dependent, coupled through linear $\left(k_{1}\right)$ and nonlinear springs $\left(k_{2}\right)$ and also through linear dashpot $\left(c_{1}\right)$ and nonlinear damping force $\left(c_{2}\right)$. In each, $m_{x}$ and $m_{y}$ mass friction forces and external forces $f_{x}$ and $f_{y}$ are applied.

The associated perpetual mechanical system without external forces is a smooth nonlinear system with unique solutions, but the existence of the solution of equations (20) depends on the external force's form on the equation's righthand side. The theorem's first condition is to examine if this mechanical system is a perpetual mechanical system. Initially, the unforced mechanical system has to be considered and eliminating the external forces (including the friction forces) in equations (20) leads to

$$
\begin{aligned}
& {\left[\begin{array}{cc}
m_{x, 0}+\dot{x}^{2} & 0 \\
0 & m_{y, 0}+\dot{y}^{2}
\end{array}\right] \times\{\ddot{x} \ddot{y}\}+\left[\begin{array}{cc}
k_{1} & -k_{1} \\
-k_{1} & k_{1}
\end{array}\right] \times\left\{\begin{array}{l}
x \\
y
\end{array}\right\}+\left[\begin{array}{cc}
c_{1} & -c_{1} \\
-c_{1} & c_{1}
\end{array}\right] \times\left\{\begin{array}{l}
\dot{x} \\
\dot{y}
\end{array}\right\}} \\
& +\left\{\begin{array}{l}
k_{2} \cdot(x-y)^{3}+c_{2} \cdot \tanh (b \cdot(\dot{x}-\dot{y})) \\
k_{2} \cdot(y-x)^{3}+c_{2} \cdot \tanh (b \cdot(\dot{y}-\dot{x}))
\end{array}\right\}=\left\{\begin{array}{l}
0 \\
0
\end{array}\right\} .
\end{aligned}
$$

In case of exact rigid body motions, 


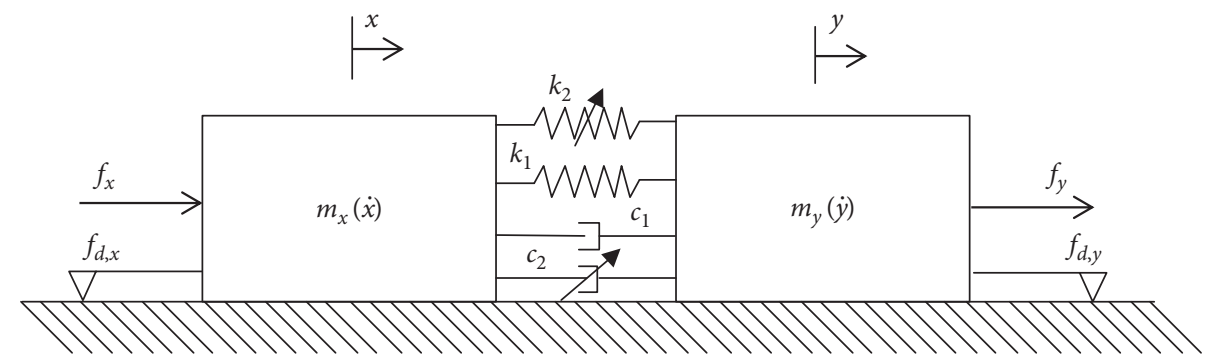

FIgUre 1: Configuration of the 2-DOF mechanical system.

$$
\begin{aligned}
& x_{a}=y_{a}, \\
& \dot{x}_{a}=\dot{y}_{a},
\end{aligned}
$$

and replacing equations (22a) and (22b) in the system of equations (21) leads to

$$
\begin{gathered}
\left(m_{x, 0}+\dot{x}_{a}^{2}\right) \cdot \ddot{x}_{a}=0 \Longleftrightarrow \ddot{x}_{a}=0, \\
\left(m_{y, 0}+\dot{y}_{a}^{2}\right) \cdot \ddot{y}_{a}=0 \Longleftrightarrow \ddot{y}_{a}=0 .
\end{gathered}
$$

The equations of jerks arise with direct differentiation of equations (21) in time, and they are given by

$$
\begin{aligned}
& {\left[\begin{array}{cc}
2 \cdot \dot{x} \cdot \ddot{x} & 0 \\
0 & 2 \cdot \dot{y} \cdot \ddot{y}
\end{array}\right] \times\{\ddot{x} \ddot{y}\}+\left[\begin{array}{cc}
m_{x, 0}+\dot{x}^{2} & 0 \\
0 & m_{y, 0}+\dot{y}^{2}
\end{array}\right] \times\left\{\begin{array}{c}
\mathrm{t} \\
x \\
\dot{t}
\end{array}\right\}+\left[\begin{array}{cc}
k_{1} & -k_{1} \\
-k_{1} & k_{1}
\end{array}\right] \times\left\{\begin{array}{l}
\dot{x} \\
\dot{y}
\end{array}\right\}+\left[\begin{array}{cc}
c_{1} & -c_{1} \\
-c_{1} & c_{1}
\end{array}\right] \times\{\ddot{x} \ddot{y}\}} \\
& \quad+\left\{3 \cdot k_{2} \cdot(x-y)^{2} \cdot(\dot{x}-\dot{y})+b \cdot c_{2} \cdot \operatorname{sech}^{2}(b \cdot(\dot{x}-\dot{y})) \cdot(\ddot{x}-\ddot{y}) 3 \cdot k_{2} \cdot(y-x)^{2} \cdot(\dot{y}-\dot{x})+b \cdot c_{2} \cdot \operatorname{sech}^{2}(b \cdot(\dot{y}-\dot{x})) \cdot(\ddot{y}-\ddot{x})\right\}=\left\{\begin{array}{l}
0 \\
0
\end{array}\right\} .
\end{aligned}
$$

Replacing equations (22a), (22b), (23a), and (23b) in equation (24) leads to

$$
\begin{aligned}
& \left(m_{x, 0}+\dot{x}^{2}\right) \cdot \stackrel{\mathfrak{t}}{x}_{a}=0 \Leftrightarrow \stackrel{\dot{t}}{x_{a}}=0, \\
& \left(m_{y, 0}+\dot{y}^{2}\right) \cdot \stackrel{\mathfrak{t}}{y}_{a}=0 \Leftrightarrow \stackrel{\mathfrak{t}}{y}_{a}=0 .
\end{aligned}
$$

The accelerations and jerks with exact rigid body motions are equal to zero, and, therefore, the system is a perpetual mechanical system.

The second condition of the theorem is that the external forcing should comply with equation (10), where, in the exact augmented perpetual manifolds (EAPMs) of this system, it is given by

$$
\begin{aligned}
& \sum_{j=1}^{2} M_{1, j}\left(\dot{x}_{a}\right)=m_{x, 0}+\dot{x}_{a}^{2}, \\
& \sum_{j=1}^{2} M_{2, j}\left(\dot{x}_{a}\right)=m_{y, 0}+\dot{x}_{a}^{2},
\end{aligned}
$$

and considering equations (26a) and (26b) with equation (9) leads to

$$
\frac{F_{x}\left(t, x_{a}, x_{a}, \dot{x}_{a}, \dot{x}_{a}\right)}{m_{x}\left(\dot{x}_{a}\right)}=\frac{F_{y}\left(t, x_{a}, x_{a}, \dot{x}_{a}, \dot{x}_{a}\right)}{m_{y}\left(\dot{x}_{a}\right)} \Longleftrightarrow F_{y}\left(t, x_{a}, x_{a}, \dot{x}_{a}, \dot{x}_{a}\right)=\frac{m_{y, 0}+\dot{x}_{a}^{2}}{m_{x, 0}+\dot{x}_{a}^{2}} \cdot F_{x}\left(t, x_{a}, x_{a}, \dot{x}_{a}, \dot{x}_{a}\right)
$$

with reference mass $m_{x}$.

The analytical solution of the external forces applied to this system is derived in Section 2.3, and it is given in Table 1. The type of vector field arising from the characteristic differential equation and the form of the external forces and the type of motion for each time interval are given in Table 2. Moreover, the equations associated with the analytical solutions, the wave velocity equations, and the associated type of motion are indicated in Table 2.
The existence of nonsmooth forces (in the third- and sixth-time intervals) requires preliminary work in developing the numerical scheme based on the switch model algorithm of [26], and herein the algorithm developed in [22] is used.

The chosen parameters for this example correspond to a car with $m_{x, 0}=2000 \mathrm{~kg}$ dragging a van with mass $m_{y, 0}=1000 \mathrm{~kg}$. The coupler has linear stiffness $k_{1}=10^{6} \mathrm{~N} / \mathrm{m}$ and nonlinear stiffness $k_{2}=5 \cdot 10^{5} \mathrm{~N} / \mathrm{m}^{3}$. In 
TABLE 2: The external forces, the solutions, and the motions in EAPMs of the 2-DOF system.

\begin{tabular}{|c|c|c|c|c|c|c|}
\hline $\mathrm{i}$ & $\begin{array}{c}\text { Time } \\
\text { interval }(s)\end{array}$ & $F_{x}^{(i)}=f_{x}+f_{d, x}$ & $\begin{array}{l}\text { Analytical } \\
\text { velocity } \dot{x}_{a, i}\end{array}$ & $\begin{array}{c}\text { Analytical } \\
\text { displacement } \\
x_{a, i}\end{array}$ & $\begin{array}{l}\text { Longitudinal } \\
\text { wave velocity } \\
w v_{i}\end{array}$ & Type of motion \\
\hline 1 & $t \in(0, t 1]$ & $\begin{array}{c}f_{x}=\sigma_{1} \cdot\left(m_{x, 0}+\dot{x}_{a}^{2}\right) \cdot f_{k, 2}^{(3)} \\
f_{d, x}=0\end{array}$ & $\begin{array}{l}\text { Equation } \\
\quad(17 \mathrm{c})\end{array}$ & Equation (18c) & Equation (19b) & $\begin{array}{l}\text { Particle linear-two } \\
\text { frequencies standing wave } \\
\text { (using specific amplitudes) }\end{array}$ \\
\hline 2 & $t \in(1, t 2]$ & $\begin{array}{c}G_{1, \mathbf{f}_{\mathbf{k}}^{(1)}}^{(1)} \\
f_{x}=\sigma_{2} \cdot\left(m_{x, 0}+\dot{x}_{a}^{2}\right) \cdot\left(f_{k}^{(3)}+f_{k}^{(1)}\right) \\
f_{d, x}=0\end{array}$ & $\begin{array}{l}\text { Equation } \\
(17 \mathrm{~d})\end{array}$ & Equation (18d) & Equation (19c) & Particle-longitudinal wave \\
\hline 3 & $t \in(2,3]$ & $\begin{array}{c}f_{y}=0 \\
f_{d, x}=\sigma_{3} \cdot\left(m_{x, 0}+\dot{x}_{a}^{2}\right) \cdot f_{k}^{(6)}\end{array}$ & $\begin{array}{l}\text { Equation } \\
(16 \mathrm{~h})\end{array}$ & Equation (16k) & $-*$ & $\begin{array}{l}\text { Particle decelerating (dry } \\
\text { friction) }\end{array}$ \\
\hline 4 & $t \in(3, t 4]$ & $\begin{array}{c}f_{x}=\sigma_{4} \cdot\left(m_{x, 0}+\dot{x}_{a}^{2}\right) \cdot f_{k}^{(5)} \\
f_{d, x}=0\end{array}$ & $\begin{array}{l}\text { Equation } \\
\quad(17 \mathrm{e})\end{array}$ & Equation (18e) & $-*$ & $\begin{array}{c}\text { Particle exponential } \\
\text { accelerating (throttle with } \\
\text { flutter) }\end{array}$ \\
\hline 5 & $t \in(4, t 5]$ & $\begin{array}{c}f_{x}=\sigma_{5} \cdot\left(m_{x, 0}+\dot{x}_{a}^{2}\right) \cdot f_{k}^{(5)} \\
f_{d, x}=0\end{array}$ & $\begin{array}{l}\text { Equation } \\
\quad(17 \mathrm{e})\end{array}$ & Equation (18e) & $-*$ & $\begin{array}{c}\text { Particle exponential } \\
\text { decelerating (throttle with } \\
\text { drag) }\end{array}$ \\
\hline 6 & $t \in(5, t 6]$ & $\begin{array}{c}f_{y}=0 \\
f_{d, x}=\sigma_{3} \cdot\left(m_{x, 0}+\dot{x}_{a}^{2}\right) \cdot f_{k}^{(6)}\end{array}$ & $\begin{array}{l}\text { Equation } \\
(16 \mathrm{p})\end{array}$ & Equation (16r) & $-*$ & $\begin{array}{l}\text { Particle decelerating (dry } \\
\text { friction) }\end{array}$ \\
\hline
\end{tabular}

${ }^{*}$ Not expected for this type of motion.

the two-degrees-of-freedom-associated linear system, the linear natural frequencies $\left(\omega_{i}\right)$ and mode shapes $\left(V_{i}\right)$ are

$$
\begin{aligned}
\omega_{1} & =0 \mathrm{rad} / \mathrm{s} \text { with normalized mode shape } \\
V_{1} & =\left\{\begin{array}{c}
0.816 \\
0.577
\end{array}\right\}, \\
\omega_{2} & =38.73 \mathrm{rad} / \mathrm{s} \text { with normalized mode shape } \\
V_{2} & =\left\{\begin{array}{c}
-0.577 \\
0.816
\end{array}\right\} .
\end{aligned}
$$

Therefore, when the second mode is excited, the two masses are vibrating in out-of-phase fashion, and although the main frequency of harmonic external forces would be this one of the second mode, this mode will not be excited due to the same direction of external forces (same sign as in equation (2)).

Considering $1 \%$ damping ratio for the second mode, the damping coefficient is $c_{1}=516.398 \mathrm{~N} \cdot \mathrm{s} / \mathrm{m}$ and the nonlinear damping coefficient has same value $c_{2}=516.398 \mathrm{~N}$ with $b=10^{6} \mathrm{~s} / \mathrm{m}$ (significantly high to approximate dry friction). In the developed theory, damping does not play any role, but the considered values are sensibly high enough, mainly for illustration purposes.

The external forcing parameters are shown in Table 3, with the associated initial conditions and the wave velocity for each type of external forcing.

All the numerical simulations in this section, with 64-bit Scilab 6.1.0 [27] using "Adams" solver with time step $\mathrm{d} t=5 \cdot 10^{-4} \mathrm{~s}$ and relative and absolute tolerance $6 \cdot 10^{-14}$, have been performed. The parameter for the convexification of the vector field in the application of the switch algorithm of [22] is $\tau=\mathrm{d} t \cdot 10^{-3}=5 \cdot 10^{-7} \mathrm{~s}$.

In Figures 2(a)-2(f), the displacements $\left(x_{a}\right)$ obtained from theoretical solution in EAPMs (equations in Table 1) and those obtained from numerical simulations of the system with equations (20) are depicted, and it seems that they are in good agreement. This can be certified further by examining the maxima's maximum of the absolute difference between the analytical and numerical displacements, which has the minimal value of $1.87510^{-8} \mathrm{~m}$.

In Figure 2(a), the displacements on the first time interval are depicted. The motion of the mechanical system is a particle standing wave with two frequencies $\left(N_{f}=2\right)$, defined by equation (h) in Table 1, and it is associated with the vector field $G_{2}^{(3)}$. In Figure 2(b), the displacements on the second time interval are depicted; the motion is a particle-longitudinal wave and is defned by equation (i) in Table 1. It is associated with the vector field $G_{1, f^{(1)}}^{(1)}$, which indicates harmonic forcing with a single frequency combined with the associated vector field $G^{(1)}$. The displacements, on the third time interval in Figure 2(c), are depicted, associated with the vector field $G^{(6)}$ for positive velocities, and they are analytically determined by equation (16k). The motion is particle decelerating due to dry friction components. In Figures 2(d)-2(e), the displacements on the fourth and fifth time intervals are depicted, and both are associated with the vector field $G^{(5)}$. They are analytically determined by equation (j) in Table 1 . In the fourth time interval (Figure $2(\mathrm{~d})$ ), the positive value of $\left(c_{d}\right)$ is associated with flutter, which indicates particle-accelerating motion, and in the fifth time interval (Figure 2(e)), the negative value of $\left(c_{d}\right)$ is associated with drag, which indicates particle-decelerating motion. Finally, in Figure 3(f), the displacements on the sixth time interval are depicted, and the motion is decelerating, and it is associated with the vector field $G^{(6)}$ for negative velocities, with displacements analytically determined by equation (16r).

In Figures 3(a)-3(f), the velocities obtained from the numerical simulations of equations (20) incorporating the analytical solutions $\left(\dot{x}_{a}\right)$ are depicted, and it seems that they are in very good agreement. Moreover, this is certified by examining the maximum of the maxima of the absolute difference between the analytical and numerically determined velocities, which has the minimal value of $1.21310^{-8} \mathrm{~m} / \mathrm{s}$. 
TABLE 3: The initial conditions, the external forcing parameters, and the wave velocities for each time interval of the 2-DOF system.

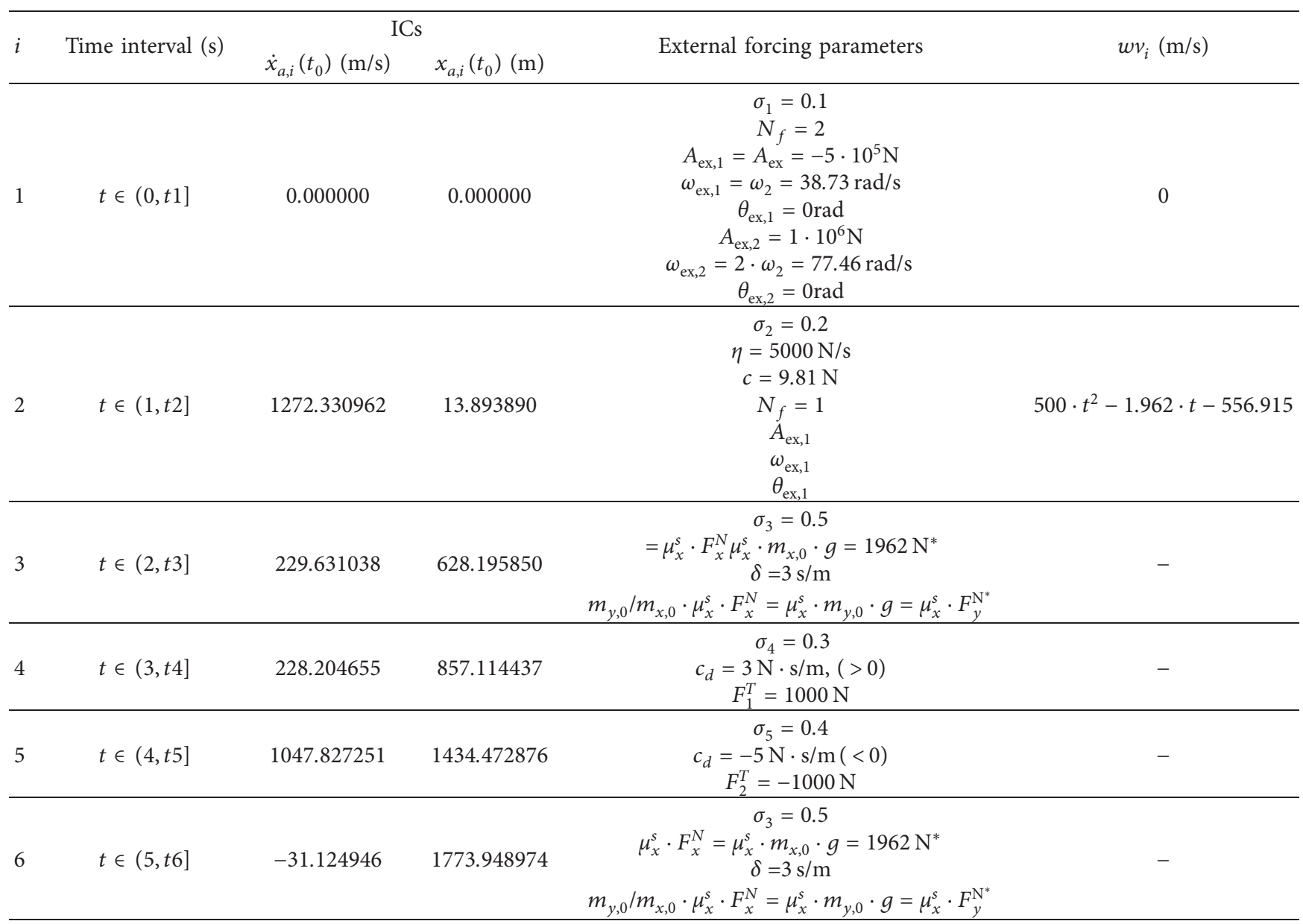

${ }^{*}$ In this example, without losing the generality and simplifying the frictional terms, the state-dependent inertia elements are considered which are not correlated with the weight of the vehicles.

In Figure 3(a), the velocities on the first time interval are depicted, with the analytical ones determined by equation (a) in Table 1 , and they are associated with the vector field $G_{2}^{(3)}$. Figure 3(b) depicts the velocities on the second time interval, and their increasing values indicate the nonzero wave velocity. The analytical velocity by equation (d) in Table 1 is given, and it is associated with the vector field $G_{1, \mathbf{f}_{\mathbf{k}}^{(1)}}^{(4)}$.

The decreasing velocity on the third time interval in Figure 3(c) is depicted, and the analytical solution by equation (16h) is determined, which is associated with the vector field $G^{(6)}$. The increasing velocities for the fourth time interval depicted in Figure 3(d) are associated with flutter. Equation (e) in Table 1 defines the analytical solution, and it is associated with the vector field $G^{(5)}$. The decreasing velocities in the fifth time interval depicted in Figure 3(e) are associated with the vector field $G^{(5)}$, for aerodynamic drag, and the analytical solution by equation (e) in Table 1 is obtained. Finally, in Figure 3(f), the velocities of the sixth time interval are depicted. They are decreasing due to dry friction, and they are associated with the vector field $G^{(6)}$. The analytical solution of the velocity on the sixth time interval by equation (16p) is determined.
The basis of each exact augmented perpetual manifold is the augmented perpetual submanifolds defined by equation (5), and it is associated with each vector field $G^{(i)}$ of the characteristic differential equation (6). In Figures 4(a)-4(f), the augmented perpetual submanifolds, defined by the analytical solutions for each time interval, are depicted. In Figure 4(a), the shape of the augmented perpetual submanifold $-\mathbf{A}_{2,0}^{(3)}$ of the vector field $-G_{2}^{(4)}$, defined by the analytical solution on the first time interval, is depicted with the first subscript indicating the number of frequencies and the second subscript indicating the wave velocity.

In Figure 4(b), using the analytical solution on the second time interval, the exact augmented perpetual submanifold $-\mathbf{A}_{1, \mathbf{f}_{k}^{(1)}}^{(4)}$ that is associated with the vector field $G_{1, f^{(1)}}^{(4)}$ is depicted, with the first subscript indicating the number of frequencies and the second subscript indicating the type of the nonharmonic function. In Figure 4(c), the exact augmented perpetual submanifold $-\mathbf{A}_{+}^{(6)}$ that is associated with the vector field $G^{(6)}$, for positive velocities indicated by the plus sign in the subscript, is depicted. The exact augmented perpetual submanifold $-\mathbf{A}_{+}^{(5)}$ that is associated with the vector field $G^{(5)}$ for flutter is depicted in 

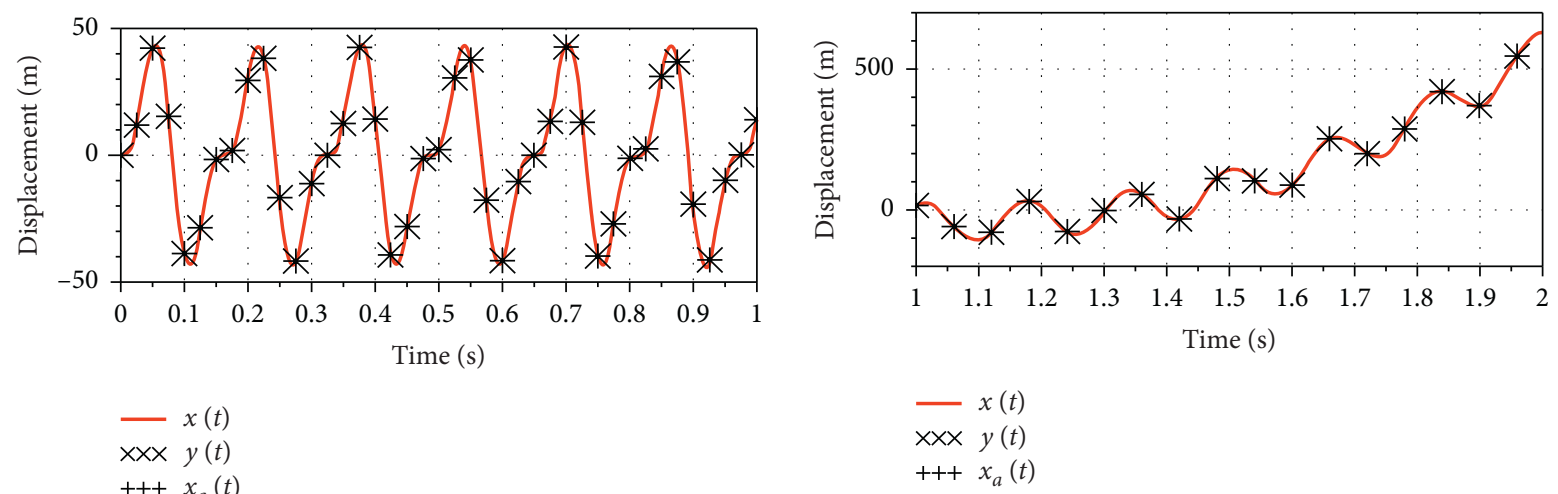

(a)

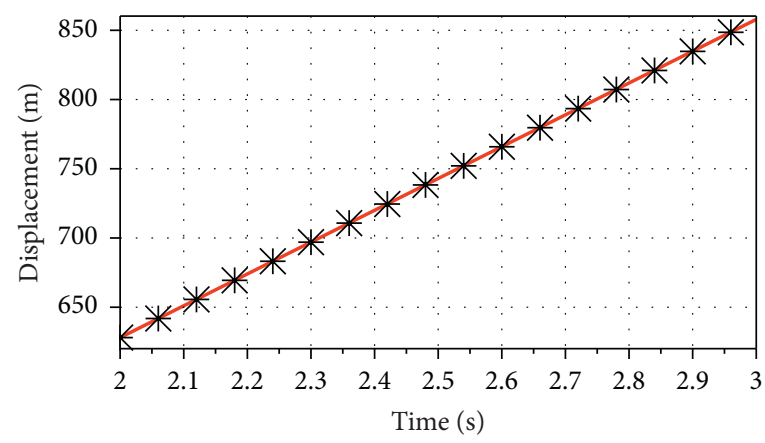

(b)

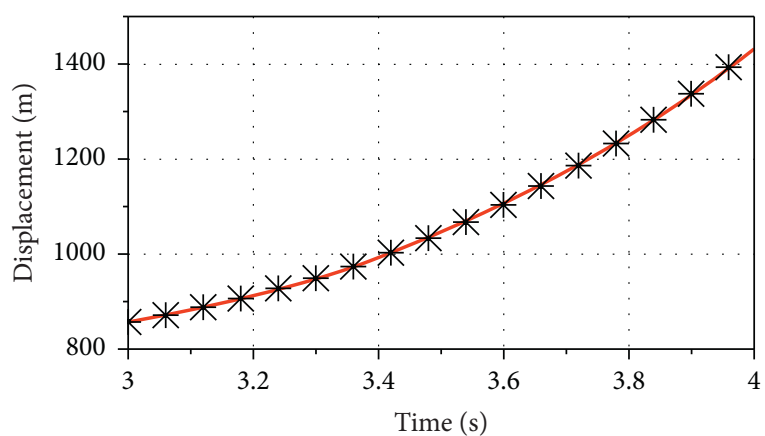

$-x(t)$
$\times \times \times \quad y(t)$
$+++\quad x_{a}(t)$

$-x(t)$

$\times \times \times \quad y(t)$

$+++x_{a}(t)$

(c)

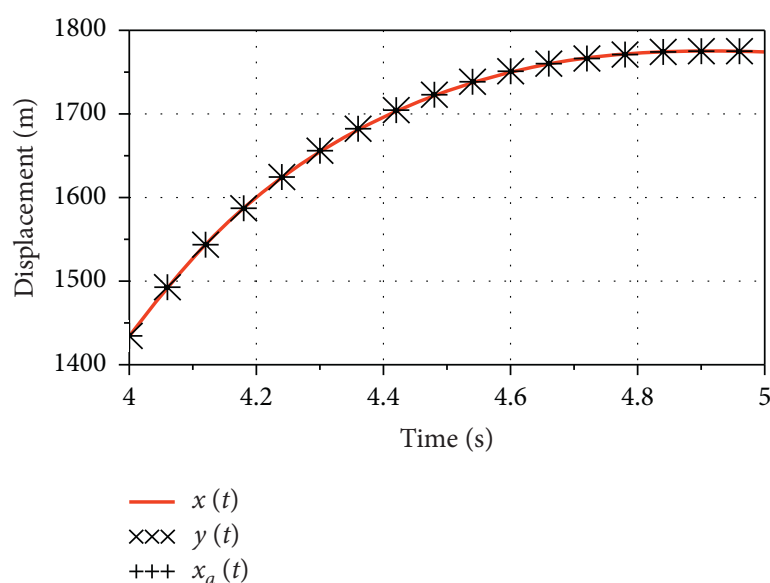

(e)

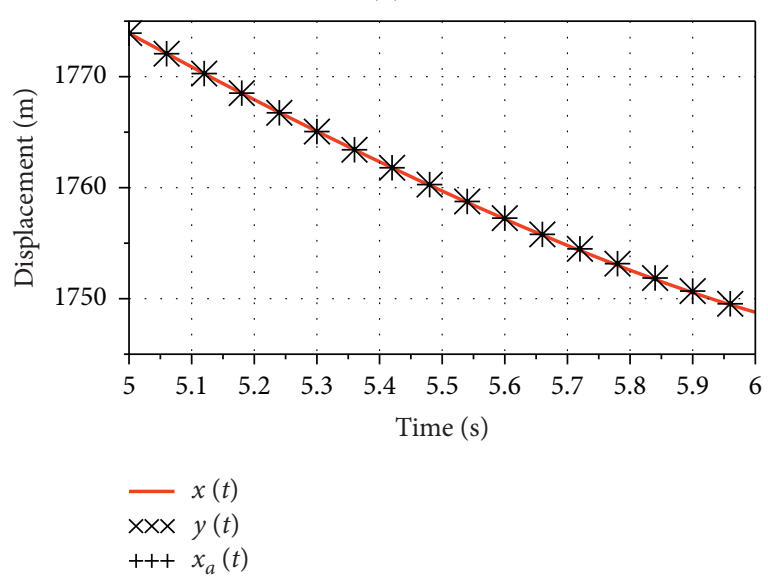

(f)

FiguRE 2: Numerically determined $(x, y)$ and analytically determined displacements $\left(x_{a}\right)$ of the 2-DOF mechanical system: (a) for the first time interval 0-1 s, with external forces $f_{k, 2}^{(3)}$; (b) for the 2nd time interval 1-2 s, with external forces $f_{k}^{(3)}$ and $f_{k}^{(1)}$; (c) for the 3 rd time interval $2-3 \mathrm{~s}$, with external forces $f_{k}^{(6)}$; (d) for the 4 th time interval 3-4 s, with external forces $f_{k}^{(5)}$; (e) for the 5th time interval 4-5 s, with external forces $f_{k}^{(5)}$; and (f) for the 6th time interval with external forces $f_{k}^{(6)}$.

Figure 4(d). In the case of aerodynamic drag, the exact augmented perpetual submanifold- $\mathbf{A}_{-}^{(5)}$ associated with the vector field $G^{(5)}$ is depicted in Figure 4(e). Finally, the exact augmented perpetual submanifold- $\mathbf{A}_{-}^{(6)}$ that is associated with the vector field $G^{(6)}$ for negative velocities is depicted in Figure 4(f).
3.2. A Shaft in Torsional Vibration with Torsional Nonlinear Energy Sink. In this example, a discretized, through finite element formulation, shaft in torsional vibration with free boundary condition in one end and dynamic boundary condition in the other end coupled with a nonlinear energy sink (NES) is considered, as indicated in Figure 5. The shaft 


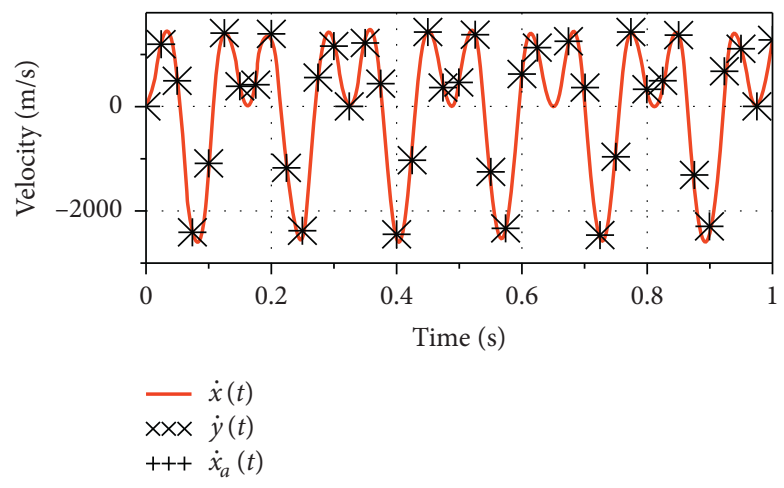

(a)

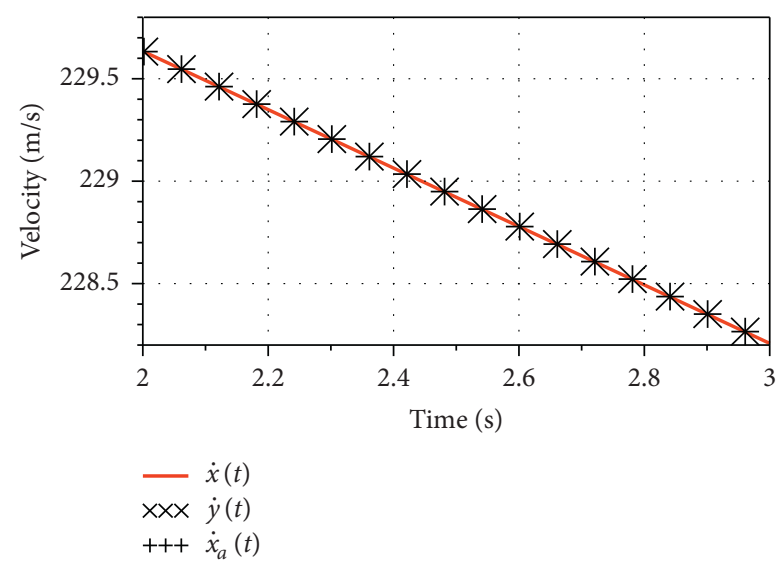

(c)

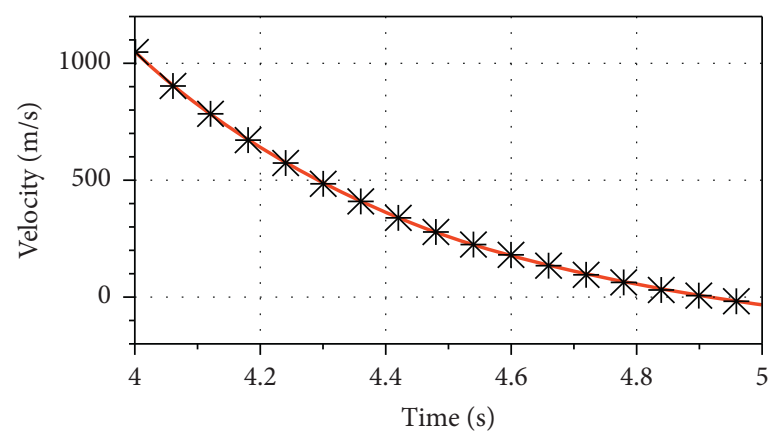

$-\dot{x}(t)$

$\times \times \times \dot{y}(t)$

$+++\dot{x}_{a}(t)$

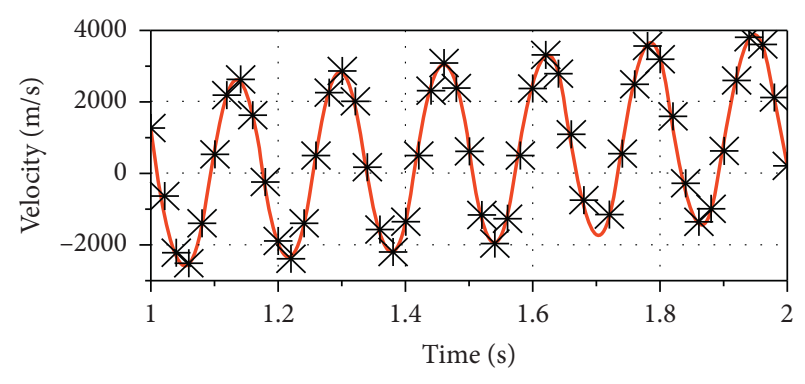

$-\dot{x}(t)$

$\times \times \times \dot{y}(t)$

$+++\dot{x}_{a}(t)$

(b)

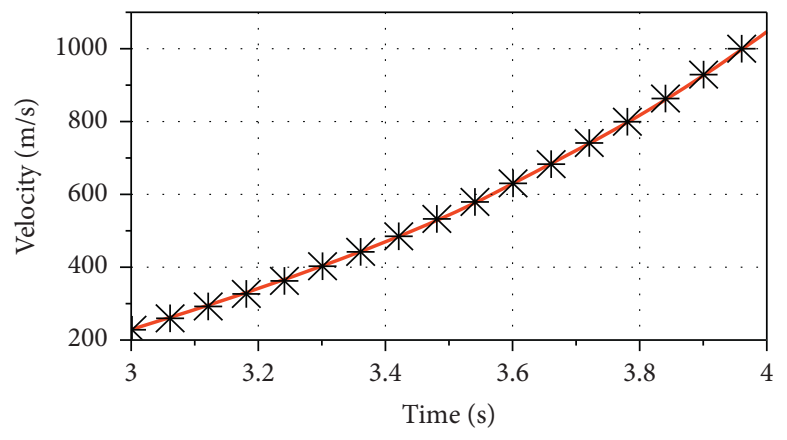

$-\dot{x}(t)$
$\times \times \times \quad \dot{y}(t)$
$+++\dot{x}_{a}(t)$

(d)

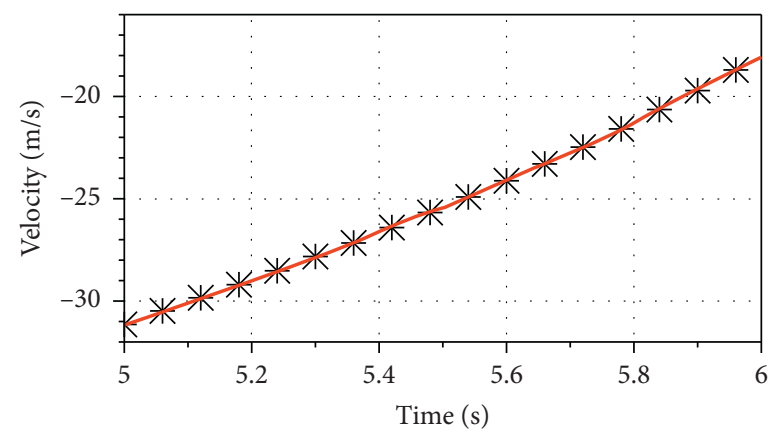

$-\dot{x}(t)$

$\mathrm{XX \times} \dot{y}(t)$

$+++\dot{x}_{a}(t)$

(e)

(f)

Figure 3: Numerically determined $(\dot{x}, \dot{y})$ and analytically determined velocities $\left(\dot{x}_{a}\right)$ of the 2-DOF mechanical system: (a) for the first time interval 0-1 s, with external forces $f_{k, 2}^{(3)}$; (b) for the 2 nd time interval 1-2 s, with external forces $f_{k}^{(3)}$ and $f_{k}^{(1)}$; (c) for the 3rd time interval 2-3 s, with external forces $f_{k}^{(6)}$; (d) for the 4 th time interval 3-4 s, with external forces $f_{k}^{(5)}$; (e) for the 5th time interval 4-5s, with external forces $f_{k}^{(5)}$; and (f) for the 6th time interval, with external forces $f_{k}^{(6)}$.

is of length $L_{\mathrm{s}}$, with internal and external diameters of $\left(D_{i}\right)$ and of $\left(D_{o}\right)$, respectively. The density is denoted as $\rho$ and the shear modulus is denoted as G. The shaft with inertia I is coupled with an NES mass, $I_{\text {nes }}$, through a linear torsional spring with stiffness $k_{1, \text { nes }}$, a nonlinear torsional spring with stiffness $k_{\text {nes }}$, and a torsional dashpot $\left(c_{\text {nes }}\right)$ as indicated in
Figure 5. The length of each element in finite element formulation is $l_{e}=L_{s} /(N)$ for all divisions of the shaft.

The discrete model of the shaft can be easily obtained. The equation of motion is described by the same partial differential equations describing the motion of rod in axial vibration $[28,29]$, and they are given by 


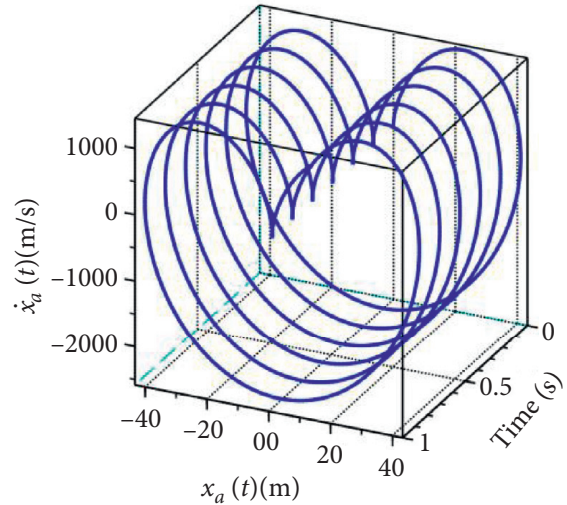

(a)

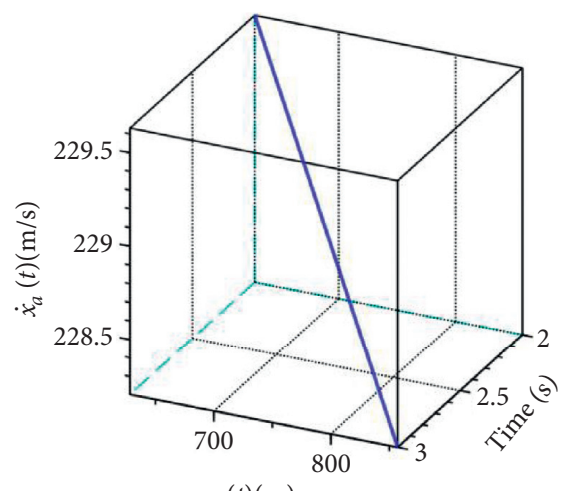

$x_{a}(t)(\mathrm{m})$

(c)

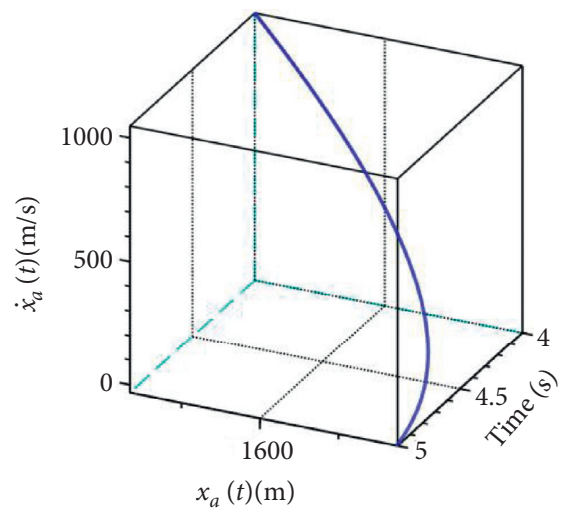

(e)

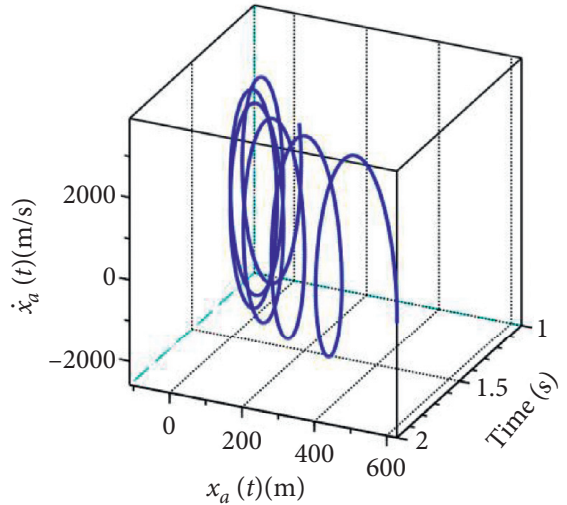

(b)

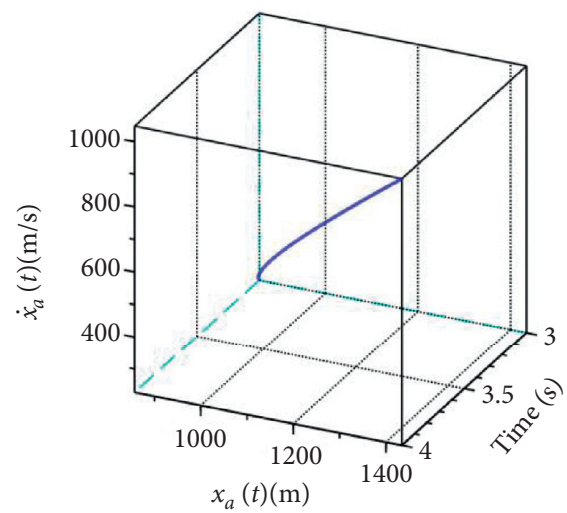

(d)

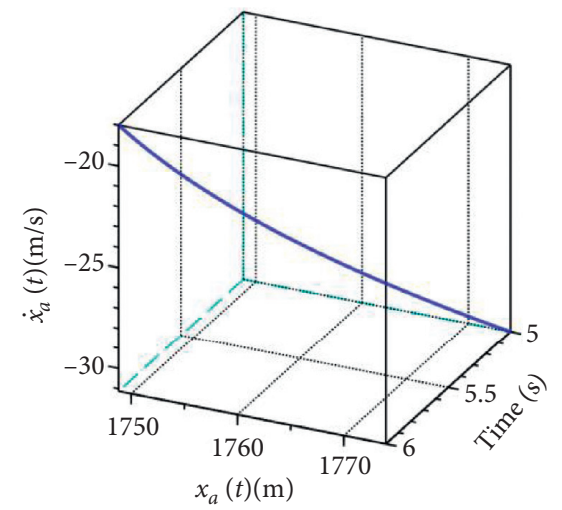

(f)

FIgURe 4: Exact augmented perpetual submanifold $\mathbf{A}^{(\mathbf{r})}$ : (a) $\mathbf{A}_{2,0}^{(3)}$ that is associated with vector field $G_{2}^{(3)}$, (b) $\mathbf{A}_{1, \mathbf{f}_{\mathrm{k}}^{(1)}}^{(4)}$ that is associated with the vector field $G_{1, \mathbf{f}_{k}^{(1)}}^{(4)}$, (c) $\mathbf{A}_{+}^{(6)}$ that is associated with the vector field $G^{(6)}$ for positive velocities, (d) $\mathbf{A}_{+}^{(5)}$ that is associated with the vector field $G^{(5)}$ for flutter, (e) $\mathbf{A}_{-}^{(5)}$ that is associated with the vector field $G^{(5)}$ for aerodynamic drag, and (f) $\mathbf{A}_{-}^{(6)}$ that is associated with the vector field $G^{(6)}$ for negative velocities.

$$
\left[I_{s}\right] \times\left\{\ddot{q}_{i}(t)\right\}+\left[C_{s}\right] \times\left\{\dot{q}_{i}(t)\right\}+\left[K_{s}\right] \times\left\{q_{i}(t)\right\}+\left\{J_{s, \mathrm{NL}, i}\right\}=\left\{J_{s, \mathrm{ex}, i}(t)\right\},
$$

with structural matrices defined explicitly in Appendix A. In case of $N$ elements discretization of the shaft, there are $N+1$ nodes in the shaft plus the NES DOF and the resulting mass, stiffness, and damping matrices dimensions being $(N+2) \times$ $(N+2)[28,29]$.

The nonlinear torque vector is given by

$$
\left\{J_{s, \mathrm{NL}, i}\right\}^{T}=k_{\mathrm{nes}} \cdot\left(q_{\mathrm{N}+1}(t)-q_{\mathrm{NES}}(t)\right)^{3} \cdot\left[\begin{array}{llll}
0 & \ldots & 1 & -1
\end{array}\right] .
$$

The external torque vector is obtained by considering only point loads at each node of the finite element formulation as follows: 


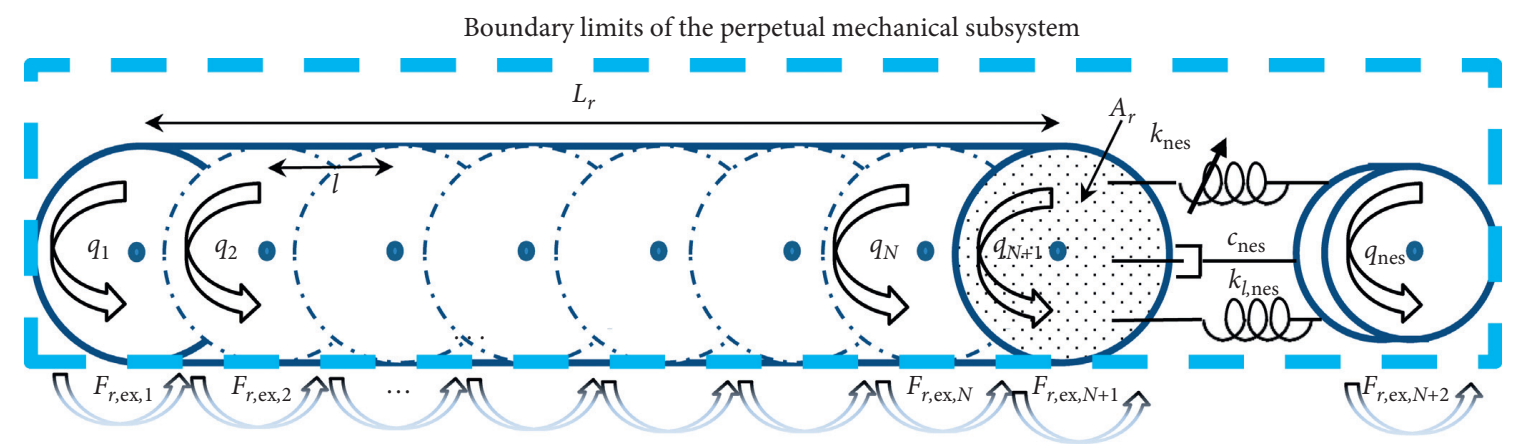

FIgURE 5: A discretized shaft in torsional vibration with NES attachment.

$$
\left\{J_{s, \mathrm{ex}, i}(t)\right\}^{T}=\left[\begin{array}{llllll}
J_{s, \mathrm{ex}, 1}(t) & J_{s, \mathrm{ex}, 2}(t) & \ldots & J_{s, \mathrm{ex}, N}(t) & J_{s, \mathrm{ex}, N+1}(t) & J_{s, \mathrm{ex}, N+2}(t)
\end{array}\right] .
$$

Initially, the mechanical system given by equation (28), by neglecting the vector forces on the right-hand side of the equations fulfilling the perpetual mechanical system's requirements, is examined. In case of rigid body motion, the following is true:

$$
\left[K_{s}\right] \times\{1\} \cdot q_{a}(t)=\left\{\sum_{j=1}^{N+2} K_{s, i, j}\right\} \cdot q_{a}(t)=\{0\},
$$

which is easily obtained by summing up all the elements of the stiffness matrix, given by equation (A.3), which belongs to each row.

Also, using equation (31) with equation (A.4) leads to

$$
\left[C_{s}\right] \times\{1\} \cdot \dot{q}_{a}(t)=\beta_{3} \cdot\left[K_{s}\right] \times\{1\} \cdot \dot{q}_{a}(t)=\{0\},
$$

$$
\left\{J_{s, \mathrm{NL}, a}\right\}^{T}=k_{\mathrm{nes}} \cdot\left(q_{a}(t)-q_{a}(t)\right)^{3} \cdot\left[\begin{array}{llll}
0 & \ldots & 1 & 1
\end{array}\right]=[0] .
$$

Therefore, taking into account equations (31)-(33) in case of unforced system, since the inertia matrix is positivedefinite, for rigid body motions, all the accelerations are equal to zero in the system of equations (28). by

The equations of jerks for the unforced system are given

$$
\left[I_{s}\right] \times\left\{\stackrel{\mathrm{t}}{q}_{i}(t)\right\}+\left[C_{s}\right] \times\left\{\ddot{q}_{i}(t)\right\}+\left[K_{s}\right] \times\left\{\dot{q}_{i}(t)\right\}+\left\{\dot{j}_{s, \mathrm{NL}, i}\right\}=\{0\},
$$

with

and

$$
\left\{\dot{j}_{s, N L, i}\right\}^{T}=3 \cdot k_{\mathrm{nes}} \cdot\left(q_{N+1}(t)-q_{\mathrm{NES}}(t)\right)^{2} \cdot\left(\dot{q}_{N+1}(t)-\dot{q}_{\mathrm{NES}}(t)\right) \cdot\left[\begin{array}{llll}
0 & \ldots & 1 & -1
\end{array}\right],
$$

where, for rigid body motion, they lead to

$$
\left\{\dot{J}_{s, \mathrm{NL}, a}\right\}^{T}=3 \cdot k_{\mathrm{nes}} \cdot\left(q_{a}(t)-q_{a}(t)\right)^{2} \cdot\left(\dot{q}_{a}(t)-\dot{q}_{a}(t)\right) \cdot\left[\begin{array}{llll}
0 & \ldots & 1 & -1
\end{array}\right]=\{0\}^{T},
$$

and, also for rigid body motions, the following is true:

$$
\left[K_{s}\right] \times\left\{\dot{q}_{a}(t)\right\}=\left[K_{s}\right] \times\{1\} \cdot \dot{q}_{a}(t)=\{0\} .
$$

Considering equations (34c) and (34d) with the fact that the accelerations are zero, in the equation that defines jerks (34a), one certifies that the jerks are equal to zero in the case of rigid body motions. Therefore, rigid body motions are acceptable perpetual manifolds for the shaft with a free end and NES attachment boundary conditions.

Summing up the rows of the inertia matrix defined by equation (A.1) leads to

$$
\begin{aligned}
\sum_{j=1}^{N+2} I_{s, 1, j} & =\sum_{j=1}^{N+2} I_{s, N+1, j}=\frac{I \cdot l_{e}}{2}, \\
\sum_{j=1}^{N+2} I_{s, k, j} & =I \cdot l_{e}, \quad \text { for } k \in\{2,3, \ldots, N\}, \\
\sum_{j=1}^{N+2} I_{s, N+2, j} & =I_{N s} .
\end{aligned}
$$

The required condition for the external torques to lead to EAPMs solution is the following: 


$$
\frac{J_{s, \mathrm{ex}, i}\left(t, q_{a}, \dot{q}_{a}\right)}{\sum_{j=1}^{N+2} I_{s, i, j}}=\frac{2 \cdot J_{s, \mathrm{ex}, 1}\left(t, q_{a}, \dot{q}_{a}\right)}{I \cdot l_{e}}=\frac{J_{s, \mathrm{exl}}\left(t, q_{a}, \dot{q}_{a}\right)}{I \cdot l_{e}}=\frac{2 \cdot J_{s, \mathrm{ex}, N+1}\left(t, q_{a}, \dot{q}_{a}\right)}{I \cdot l_{e}}=\frac{J_{s, \mathrm{ex}, N+2}\left(t, q_{a}, \dot{q}_{a}\right)}{I_{\mathrm{nes}}}, \quad \text { for } i \in\{1,2, \ldots, N+2\} \text {, and } l \in\{2,3, \ldots, N\}
$$

which leads to several arrangements of the external torques and herein the following is considered:

$$
\begin{aligned}
J_{s, \mathrm{ex}, 1}\left(t, q_{a}, \dot{q}_{a}\right) & =J_{s, \mathrm{ex}, N+1}\left(t, q_{a}, \dot{q}_{a}\right), \\
J_{s, \mathrm{ex}, l} & =2 \cdot J_{s, \mathrm{ex}, 1}\left(t, q_{a}, \dot{q}_{a}\right)=2 \cdot J_{s, \mathrm{ex}, 1}\left(t, q_{a}, \dot{q}_{a}\right), \quad \text { for } l \in\{2,3, \ldots, N\}, \\
J_{s, \mathrm{ex}, N+2} & =\frac{2 \cdot I_{\mathrm{nes}}}{I \cdot l_{e}} \cdot J_{s, \mathrm{ex}, 1}\left(t, q_{a}, \dot{q}_{a}\right)=\frac{2 \cdot I_{\mathrm{nes}}}{I \cdot l_{e}} \cdot J_{s, \mathrm{ex}, 1}\left(t, q_{a}, \dot{q}_{a}\right) .
\end{aligned}
$$

Considering the system of equations (28) and the external torques defined by equations $(37 a)-(37 c)$, the solution is given by solving the following characteristic differential equation:

$$
\ddot{q}_{a}(t)=\frac{J_{s, \mathrm{ex}, i}\left(t, q_{a}, \dot{q}_{a}\right)}{\sum_{j=1}^{N} I_{s, i, j}}=\frac{J_{s, \mathrm{ex}, 1}}{I \cdot l_{e}} .
$$

Four different types of excitation forces are applied in the shaft, as is shown in Table 4. Also, in Table 4, the equations that determine the shaft's motion for each time interval, the wave velocity where it is applicable, and the type of motion are given. Of the first, second, and fourth time intervals, the external forces are the same as those applied in the train as 5DOF mechanical system model in [22].

A stainless-steel solid shaft with external $r_{o}=0.03 \mathrm{~m}$, length of $L=1 \mathrm{~m}$, density of $\rho_{0}=8000 \mathrm{~kg} / \mathrm{m}^{3}$, and shear modulus of $G=76.9 \mathrm{GPa}$, is considered. The geometrical and material properties of the shaft lead to an inertia coefficient $I=5.0894 \cdot 10^{-3} \mathrm{~kg} / \mathrm{m}^{3}$. The NES inertia coefficient is $I_{\mathrm{NES}}=5.0894 \cdot 10^{-4} \mathrm{~kg} / \mathrm{m}^{2}$, linear torsional stiffness is $k_{l, \text { nes }}=2000 \mathrm{~N} \cdot \mathrm{m} / \mathrm{rad}$, and nonlinear torsional stiffness is $k_{\text {nes }}=2000 \mathrm{~N} \cdot \mathrm{m} / \mathrm{rad}^{3}$. The number of elements for the discretization of the shaft is $N=100$. The first three natural frequencies of the underlying linear system, which are obtained through the eigenvalue solution, are $\omega_{s, 1}=0 \mathrm{rad} / \mathrm{sec}$, $\omega_{s, 2}=2064.67 \mathrm{rad} / \mathrm{sec}$, and $\omega_{s, 3}=9782.50 \mathrm{rad} / \mathrm{sec}$. The Rayleigh damping coefficients are $\alpha=0 s^{-1}$ and $\beta_{1}=9.686798$. $10^{-6} \mathrm{~s}$ which correspond to $1 \%$ damping ratio of the second natural frequency of the underlying linear system.

The values of the external forcing parameters are given in Table 5. Also, in this table, the initial conditions resulting from previous time interval motion associated with each time interval as well as the wave velocities are shown. After the first time interval, the initial conditions from the previous interval motion are given.

All the numerical simulations in this section have been performed with 64-bit Scilab 6.6.1 [27] using “Adams" solver with time step $7.6080 \cdot 10^{-6} \mathrm{~s}$, as well as relative and absolute tolerance $5 \cdot 10^{-15}$.

In Figures 6(a)-6(d), selected numerically determined angular displacements of the mechanical system, of the left edge of the shaft $\left(q_{1}\right)$, of the NES $\left(q_{102}\right)$, and of the middle $\left(q_{50}\right)$ of the shaft are depicted, incorporating the analytical solution $\left(q_{a}\right)$. They seem to be in good agreement and this can be certified further by considering the maximum of the maxima of the absolute differences between the analytical solution and each angular displacement of the mechanical system, which has the minimal value of $1.99910^{-10} \mathrm{rad}$. In Figure 6(a), the angular displacements for the first time interval are depicted, which is a particle standing wave solution, and they are associated with the vector field $G^{(2)}$ with analytical solution given by equation (g) in Table 1 and it has the same solution of the train example on the second time interval in [22]. The angular displacements for the second time interval in Figure 6(b) are depicted; they have particle curvilinear function motion, and they are associated with the vector field $G^{(1)}$. The analytical solution is given by equation (f) in Table 1, and it has the same solution as the train example of [22] for the first time interval. In Figure 6(c), the angular displacements for the third time interval are depicted, which correspond to particle-motion directed two-frequency wave, and they are associated with the vector field $G_{2}^{(3)}$. The analytical solution is given by equation (h) in Table 1 considering two frequencies of the external forcing. In Figure 6(d) the angular displacements for the fourth time interval are depicted, corresponding to particle-motion directed single-frequency wave and associated with the vector field $G^{(2)}$. The analytical solution of displacements by equation ( $\mathrm{g}$ ) in Table 1 is given, and it has the same solution as the train example for the third time interval in [22].

In Figures $7(\mathrm{a})-7(\mathrm{~d})$, selected numerically determined angular velocities (the same ones depicted in the previous Figure 6) are depicted, incorporating the analytically 
TABLE 4: Equations that describe the solutions in augmented perpetual manifolds with the expected motions at different time intervals $\left(T_{\mathrm{ch}}=1.52159874 \cdot 10^{-2} \mathrm{~s}\right)$.

\begin{tabular}{|c|c|c|c|c|c|c|}
\hline $\mathrm{i}$ & Time interval (s) & $G^{(r)}=J_{s, \mathrm{ex}, 1}^{(r)} / I \cdot l_{e}$ & $\begin{array}{l}\text { Analytical } \\
\text { velocity } \dot{q}_{a, i}\end{array}$ & $\begin{array}{c}\text { Analytical } \\
\text { displacement } q_{a, i}\end{array}$ & $\begin{array}{l}\text { Longitudinal wave } \\
\text { velocity } w v_{i}\end{array}$ & Type of motion \\
\hline 1 & $t \in\left(0, \mathrm{~T}_{c h}\right]$ & $\begin{array}{c}G^{(2)}=f_{k}^{(2)} / I \cdot l_{e}, \text { Equation } \\
(11)\end{array}$ & Equation (17b) & Equation (18b) & Equation (19a) & $\begin{array}{c}\text { Particle-standing } \\
\text { wave }\end{array}$ \\
\hline 2 & $t \in\left(T_{\mathrm{ch}}, 2 \cdot T_{\mathrm{ch}}\right]$ & $\begin{array}{c}G^{(1)}=f_{k}^{(1)} / I \cdot l_{e}, \text { Equation } \\
(10)\end{array}$ & Equation (17a) & Equation (18a) & $-^{*}$ & Particle curvilinear \\
\hline 3 & $t \in\left(2 \cdot T_{\mathrm{ch}}, 3 \cdot T_{\mathrm{ch}}\right]$ & $\begin{array}{c}G_{2}^{(3)}=f_{k, 2}^{(3)} / I \cdot l_{e}, \text { Equation } \\
(12 \mathrm{a})\end{array}$ & Equation (17c) & Equation (18c) & Equation (19b) & $\begin{array}{l}\text { Particle-motion } \\
\text { directed wave }\end{array}$ \\
\hline 4 & $t \in\left(3 \cdot T_{\mathrm{ch}}, 4 \cdot T_{\mathrm{ch}}\right]$ & $\begin{array}{c}G^{(2)}=f_{k}^{(2)} / I \cdot l_{e}, \text { Equation } \\
(11)\end{array}$ & Equation (17b) & Equation (18b) & Equation (19a) & $\begin{array}{l}\text { Particle-motion } \\
\text { directed wave }\end{array}$ \\
\hline
\end{tabular}

${ }^{*}$ Not applicable on this type of motion.

TABle 5: External forcing parameters of the shaft with the associated initial conditions for each time interval and the theoretical wave velocity.

\begin{tabular}{|c|c|c|c|c|c|}
\hline \multirow{2}{*}{$i$} & \multirow{2}{*}{ Time interval (s) } & \multicolumn{2}{|c|}{ ICs } & \multirow{2}{*}{ External forcing parameters } & \multirow{2}{*}{$w v_{i}(t)(\mathrm{rad} / \mathrm{s})$} \\
\hline & & $\dot{q}_{a, i}\left(t_{0}\right)(\mathrm{rad} / \mathrm{s})$ & $q_{a, i}\left(t_{0}\right)(\mathrm{rad})$ & & \\
\hline 1 & $t \in(0,0.0152]$ & -190.333562 & 0.000000 & $\begin{aligned} A_{\mathrm{ex}, 1} & =10 \mathrm{~N} \cdot \mathrm{m} \\
\omega_{\mathrm{ex}, 1}=\omega_{s, 2} & =2064.67 \mathrm{rad} / \mathrm{s} \\
\theta_{\mathrm{ex}} & =0 \mathrm{rad}\end{aligned}$ & 0.00 \\
\hline 2 & $t \in(0.01520,0.0304]$ & -190.333562 & 0.000000 & $\begin{array}{c}\eta=1.5 \mathrm{~N} \cdot \mathrm{m} / \mathrm{s} \\
c=1 \mathrm{~N} / \mathrm{m}\end{array}$ & - \\
\hline 3 & $t \in(0.0304,0.0456]$ & 428.088376 & 1.791532 & $\begin{array}{c}A_{\mathrm{ex}, 1} \\
\omega_{\mathrm{ex}, 1} \\
\theta_{\mathrm{ex}} \\
A_{\mathrm{ex}, 1} \\
\omega_{\mathrm{ex}, 2}=2 \cdot \omega_{s, 2}=4129.33 \mathrm{rad} / \mathrm{s} \\
\theta_{\mathrm{ex}}\end{array}$ & 713.59 \\
\hline 4 & $t \in(0.0456,0.0609]$ & 428.088376 & 12.649489 & $\begin{array}{c}A_{\mathrm{ex}, 1} \\
\omega_{\mathrm{ex}, 1} \\
\theta_{\mathrm{ex}}\end{array}$ & 618.42 \\
\hline
\end{tabular}

determined angular velocities, and they seem to be in very good agreement. This is certified by examining the maximum of the maxima of the absolute differences of the analytical and each of the numerically determined velocities, which has the minimal value of $1.47910^{-8} \mathrm{rad} / \mathrm{s}$.

In Figure $7(\mathrm{a})$, the angular velocities in the first time interval are depicted, and they are analytically determined by equation (b) in Table 1 . The angular velocities for the second time interval in Figure 7(b) are depicted, and they are analytically determined by equation (a) in Table 1 . In Figure $7(\mathrm{c})$, the angular velocities for the third time interval are depicted, and they are analytically determined by equation (c) in Table 1. The angular velocities for the last time interval in Figure $7(\mathrm{~d})$ are depicted by equation (b) in Table 1.

In Figures $8(a)-8(d)$, the rest of exact augmented perpetual submanifolds defined by the vector fields of Table 1 , which are not determined in the 2-DOF example of Section 3.1, are depicted. Figure 8(a) depicts the exact augmented perpetual submanifold $\mathbf{A}_{2,0}^{(2)}$ that is associated with the vector field $G^{(2)}$ for particle-standing wave motion, and it has the same form as the train example for the second time interval of [22]. The exact augmented perpetual submanifold $\mathbf{A}^{(1)}$ in Figure $8(\mathrm{~b})$, which is associated with the vector field $G^{(1)}$, is depicted, and it has the same form as the train example for the first time interval of [22]. In Figure 8(c), the exact augmented perpetual submanifold $\mathbf{A}_{2}^{(3)}$ that is associated with the vector field $G_{2}^{(3)}$ is depicted. The exact augmented perpetual submanifold $\mathbf{A}_{2}^{(2)}$ that is associated with vector field $G^{(2)}$ is depicted in Figure 8(d), and it has the same form as the train example of [22] for the third time interval.

3.3. A Five-Degrees-of-Freedom System. In this section, the train modeled as a five-degrees-of-freedom system in [22] is considered, and the motion is described by the following equation of motion:

$$
\left[\mathbf{M}_{\mathbf{i}, \mathbf{j}}\right] \times\left\{\ddot{x}_{i}\right\}+\left[\mathbf{C}_{\mathbf{i}, \mathbf{j}}\right] \times\left\{\dot{x}_{i}\right\}+\left[\mathbf{K}_{\mathbf{i}, \mathbf{j}}\right] \times\left\{x_{i}\right\}+\left\{F_{i}^{N L}\left(x_{n}, \dot{x}_{o}\right)\right\}=\left\{F_{\text {ext }, i}(t)\right\}, \quad \text { for } n, o \in\{1,2, \ldots, 5\}, i=1, \ldots, 5 \text {, }
$$



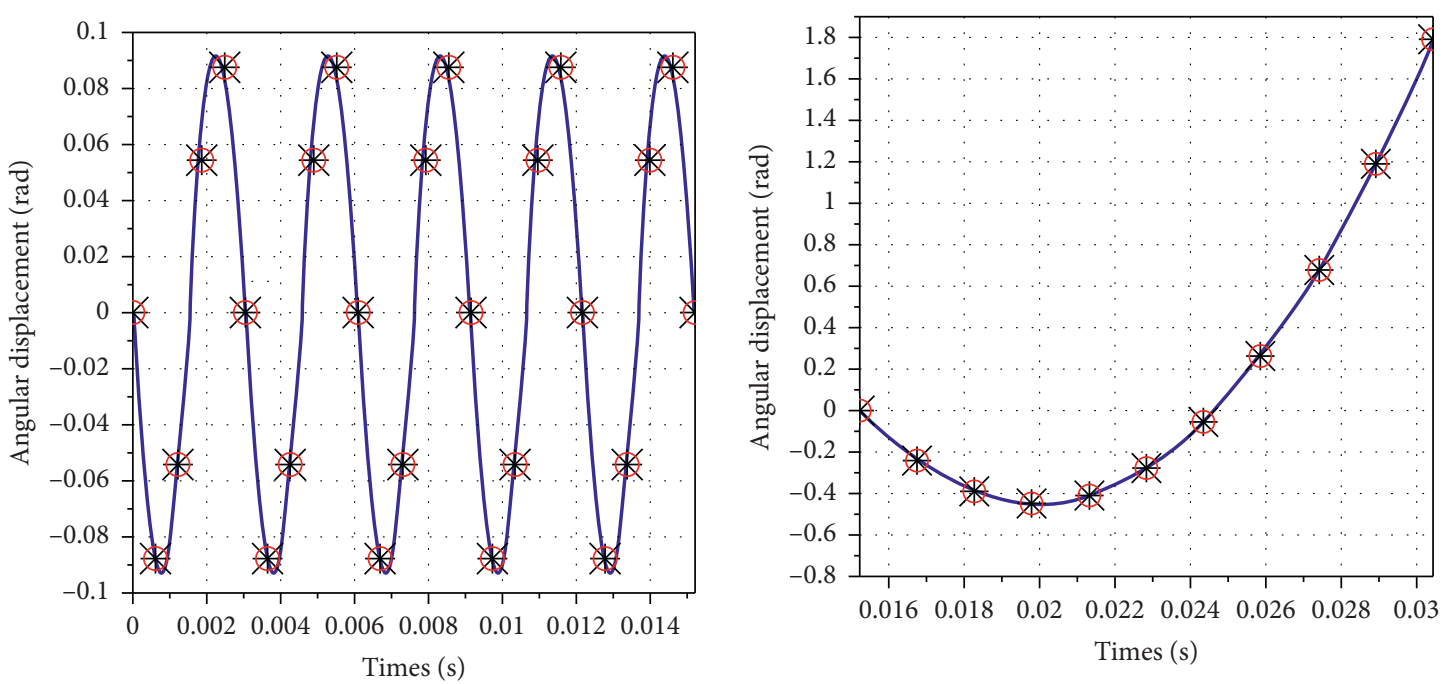

$$
\begin{array}{ll} 
& q_{1}(t) \\
\times \times \times & q_{50}(t) \\
+++ & q_{102}(t) \\
\infty & q_{a}(t)
\end{array}
$$

(a)

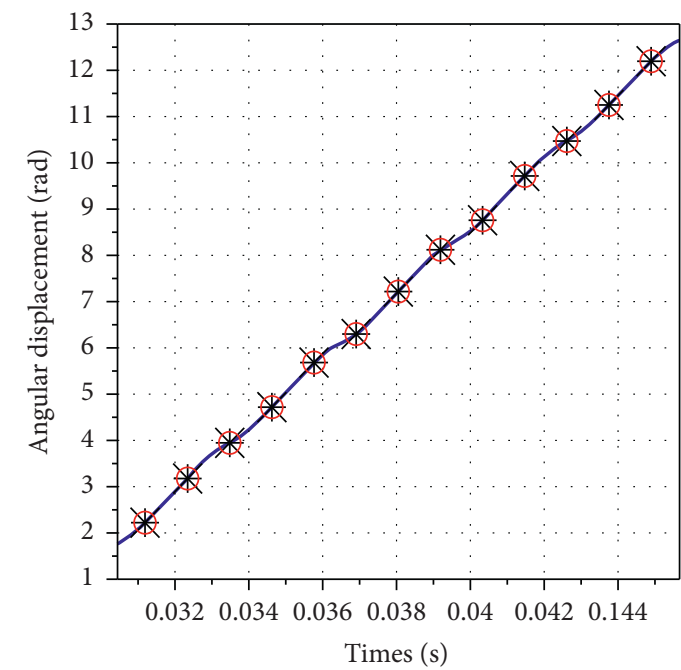

$$
\begin{array}{ll}
- & q_{1}(t) \\
\times \times \times & q_{50}(t) \\
+++ & q_{102}(t) \\
\infty & q_{a}(t)
\end{array}
$$

(b)

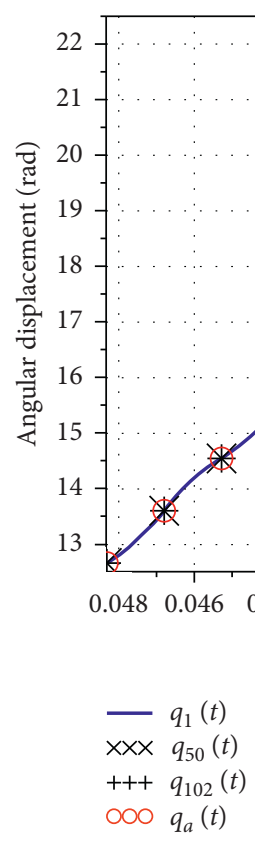

(c)

(d)

FIGURE 6: Selected numerically determined angular displacements of the FE model, of the left edge of the shaft $\left(q_{1}\right)$, of the NES $\left(q_{102}\right)$, and of the middle $\left(q_{50}\right)$ of the shaft, incorporating the analytical solution $\left(q_{a}\right)$ : (a) for the first time interval 0-1 s, with external forces $f_{k}^{(2)}$; (b) for the 2nd time interval 1-2 s, with external forces $f_{k}^{(1)} ;$ (c) for the 3rd time interval 2-3 s, with external forces $f_{k}^{(3)}$; and (d) for the 4 th time interval 3-4 s, with external forces $f_{k}^{(2)}$. 

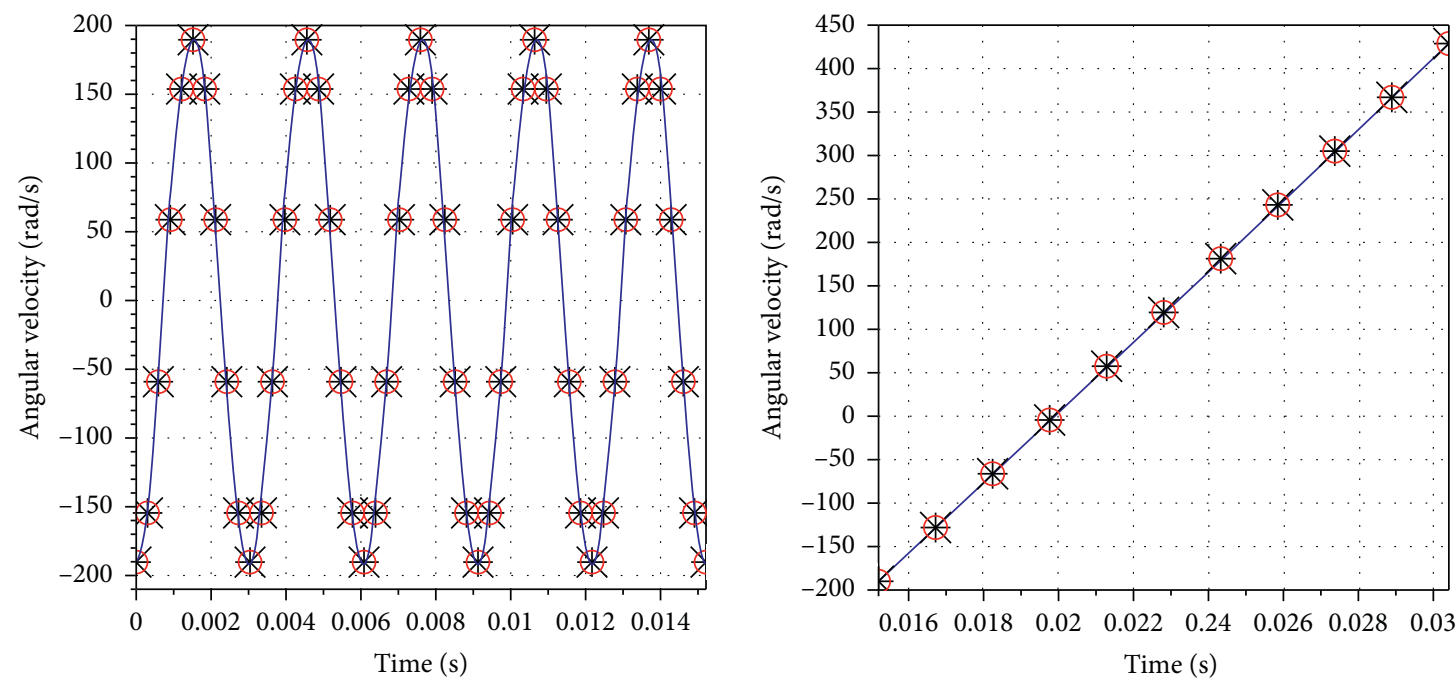

$$
\begin{array}{ll} 
& \dot{q}_{1}(t) \\
\times \times \times & \dot{q}_{50}(t) \\
+++ & \dot{q}_{102}(t) \\
\infty & \dot{q}_{a}(t)
\end{array}
$$

$$
\begin{aligned}
& \text { - } \dot{q}_{1}(t) \\
& \text { XXX } \dot{q}_{50}(t) \\
& +++\dot{q}_{102}(t) \\
& \text { ○০০ } \dot{q}_{a}(t)
\end{aligned}
$$

(a)

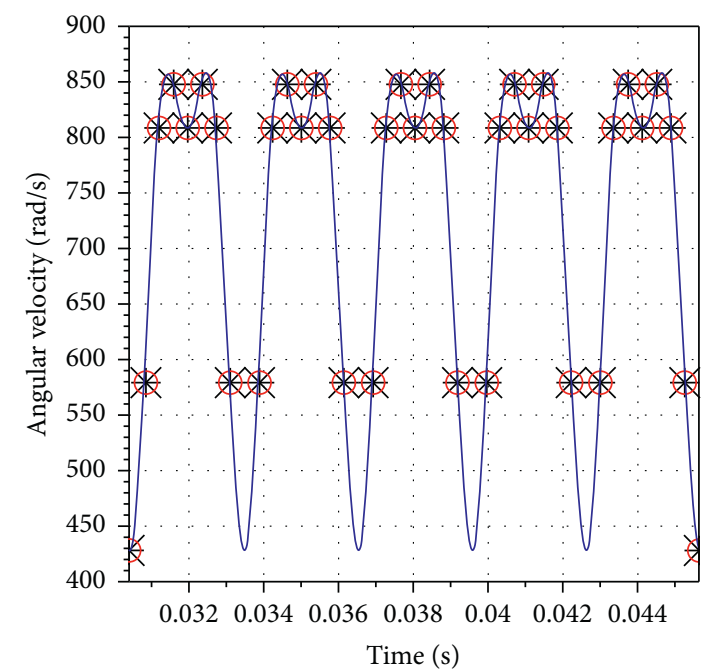

(b)

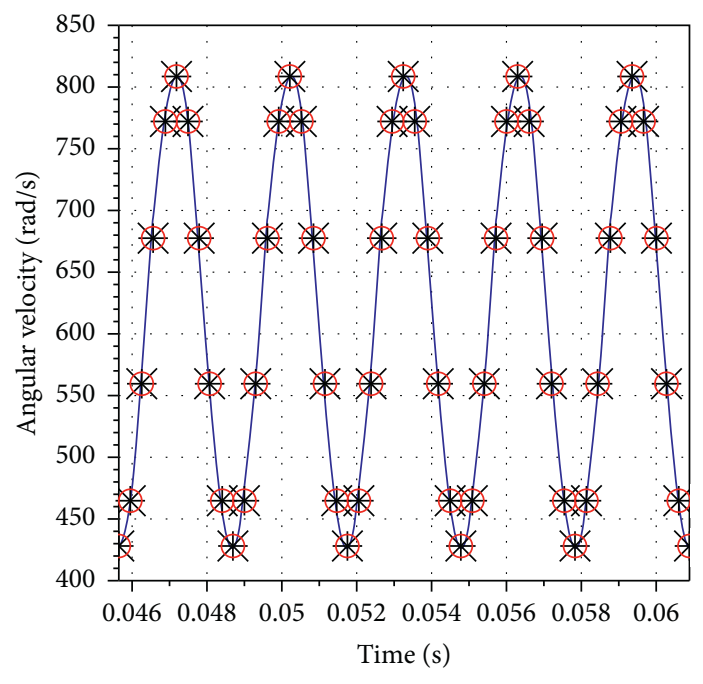

$$
\begin{array}{ll} 
& \dot{q}_{1}(t) \\
\times \times \times & \dot{q}_{50}(t) \\
+++ & \dot{q}_{102}(t) \\
\text { ○๐ } & \dot{q}_{a}(t)
\end{array}
$$

$$
\begin{array}{ll}
- & \dot{q}_{1}(t) \\
\times \times \times & \dot{q}_{50}(t) \\
+++ & \dot{q}_{102}(t) \\
\text { ○○ } & \dot{q}_{a}(t)
\end{array}
$$

(c)

(d)

FIGURE 7: Selected numerically determined angular velocities of the FE model, of the left edge of the shaft $\left(q_{1}\right)$, of the NES $\left(q_{102}\right)$, and of the middle $\left(q_{50}\right)$ of the shaft, incorporating the analytical solution $\left(q_{a}\right)$ : (a) for the first time interval 0-1 s, with external forces $f_{k}^{(2)}$; (b) for the 2nd time interval 1-2 s, with external forces $f_{k}^{(1)}$; (c) for the 3rd time interval 2-3 s, with external forces $f_{k}^{(3)}$; and (d) for the 4 th time interval 3-4 s, with external forces $f_{k}^{(2)}$. 


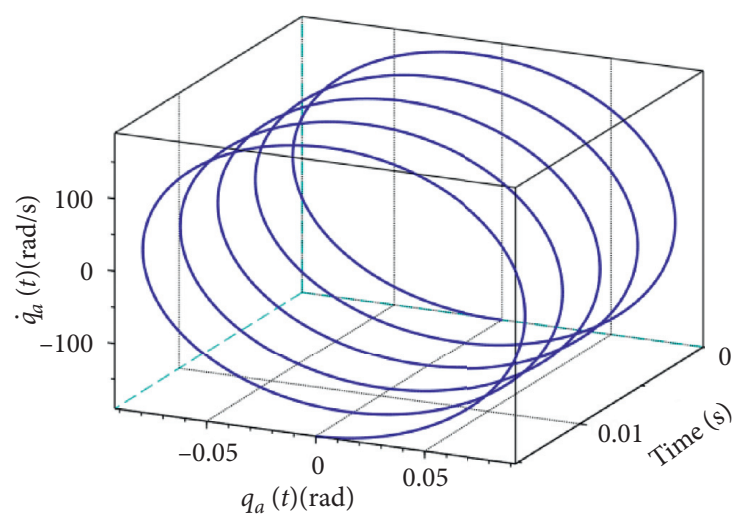

(a)

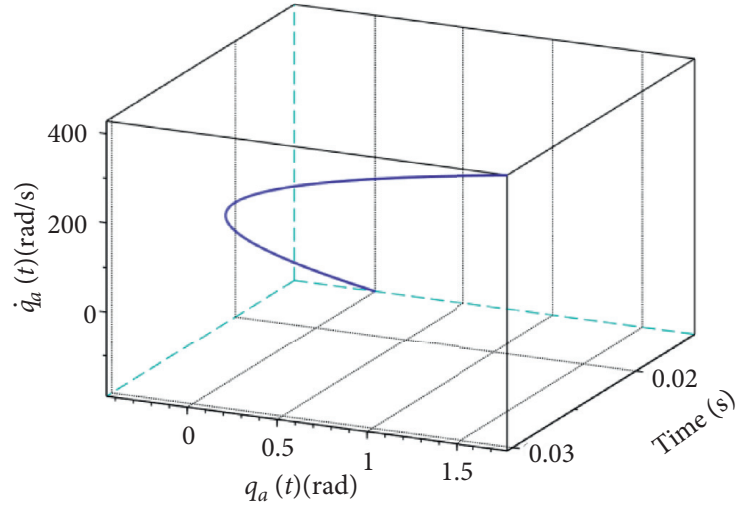

(b)

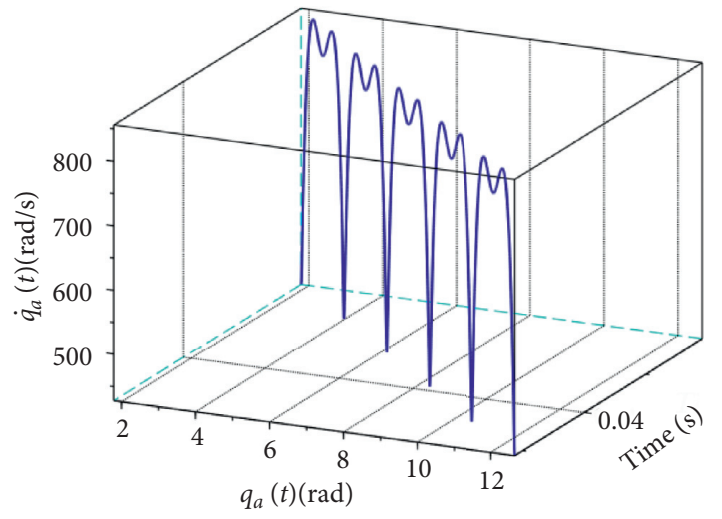

(c)

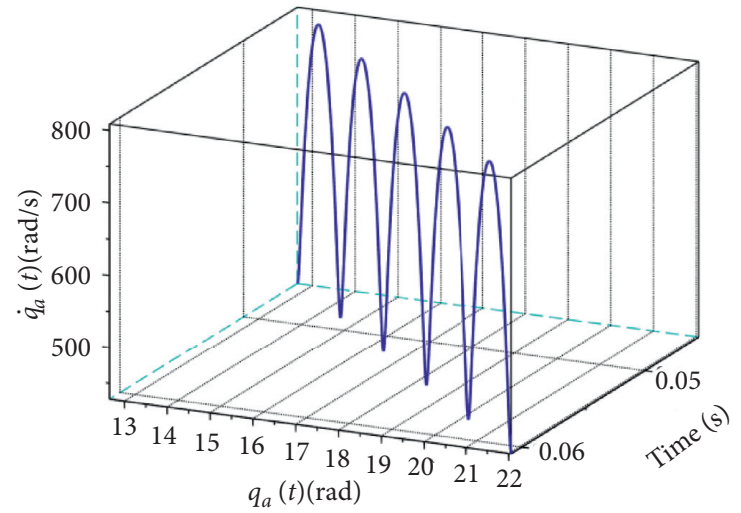

(d)

Figure 8: Exact augmented perpetual submanifolds $\mathbf{A}^{(\mathbf{r})}$ : (a) $\mathbf{A}_{2,0}^{(2)}$ that is associated with the vector field $\mathbf{G}^{(2)}$, (b) $\mathbf{A}^{(1)}$ that is associated with the vector field $\mathbf{G}^{(1)}$, (c) $\mathbf{A}_{2}^{(3)}$ that is associated with the vector field $\mathbf{G}_{2}^{(3)}$, and (d) $\mathbf{A}_{2}^{(2)}$ that is associated with the vector field $\mathbf{G}^{(2)}$. 
with mass matrix defined by

$$
\left[\mathbf{M}_{\mathbf{i}, \mathbf{j}}\right]=\left[\begin{array}{ccccc}
m_{1} & 0 & 0 & 0 & 0 \\
0 & m_{2} & 0 & 0 & 0 \\
0 & 0 & m_{3} & 0 & 0 \\
0 & 0 & 0 & m_{4} & 0 \\
0 & 0 & 0 & 0 & m_{5}
\end{array}\right]
$$

and $m_{i}(i=1, \ldots, 5)$ being positive constants; therefore all sums of rows are nonzero.

The stiffness matrix is defined by

$$
\left[\mathbf{K}_{\mathbf{i}, \mathbf{j}}\right]=\left[\begin{array}{ccccc}
k_{1} & -k_{1} & 0 & 0 & 0 \\
-k_{1} & k_{1}+k_{2} & -k_{2} & 0 & 0 \\
0 & -k_{2} & k_{2}+k_{3} & -k_{3} & 0 \\
0 & 0 & -k_{3} & k_{3}+k_{4} & -k_{4} \\
0 & 0 & 0 & -k_{4} & k_{4}
\end{array}\right] .
$$

The damping matrix is defined with Rayleigh damping and given by

$$
\left[\mathbf{C}_{\mathbf{i}, \mathbf{j}}\right]=\left[\begin{array}{ccccc}
c_{1} & -c_{1} & 0 & 0 & 0 \\
-c_{1} & c_{1}+c_{2} & -c_{2} & 0 & 0 \\
0 & -c_{2} & c_{2}+c_{3} & -c_{3} & 0 \\
0 & 0 & -c_{3} & c_{3}+c_{4} & -c_{4} \\
0 & 0 & 0 & -c_{4} & c_{4}
\end{array}\right]=\beta \cdot\left[\mathbf{K}_{\mathbf{i}, \mathbf{j}}\right] .
$$

The nonlinear forces vector is given by

$$
\left\{F_{i}^{\mathrm{NL}}\left(x_{n}, \dot{x}_{o}\right)\right\}=\left\{\begin{array}{c}
k_{n l, 1} \cdot \sin \left(x_{1}-x_{2}\right)+c_{n l, 1} \cdot\left(\dot{x}_{1}-\dot{x}_{2}\right)^{3} \\
-k_{n l, 1} \cdot \sin \left(x_{1}-x_{2}\right)-c_{n l, 1} \cdot\left(\dot{x}_{1}-\dot{x}_{2}\right)^{3}+k_{n l, 2} \cdot\left(x_{2}-x_{3}\right)^{5}+c_{n l, 2} \cdot\left(\dot{x}_{2}-\dot{x}_{3}\right)^{5} \\
-k_{n l, 2} \cdot\left(x_{2}-x_{3}\right)^{5}-c_{n l, 2} \cdot\left(\dot{x}_{2}-\dot{x}_{3}\right)^{5}+k_{n l, 3} \cdot \sin \left(x_{3}-x_{4}\right)+c_{n l, 3} \cdot\left(\dot{x}_{3}-\dot{x}_{4}\right)^{7} \\
-k_{n l, 3} \cdot \sin \left(x_{3}-x_{4}\right)-c_{n l, 3} \cdot\left(\dot{x}_{3}-\dot{x}_{4}\right)^{7}+k_{n l, 4} \cdot\left(x_{4}-x_{5}\right)^{7}+c_{n l, 4} \cdot \tanh \left(b \cdot\left(\dot{x}_{4}-\dot{x}_{5}\right)\right) \\
-k_{n l, 4} \cdot\left(x_{4}-x_{5}\right)^{7}-c_{n l, 4} \cdot \tanh \left(b \cdot\left(\dot{x}_{4}-\dot{x}_{5}\right)\right)
\end{array}\right\}, \quad \text { for } n, o \in\{1,2, \ldots, 5\}
$$

and the theorem's condition for the external forcing vector (equation (9)), considering the first mass as reference $k$ mass, leads to

$$
\left\{F_{\mathrm{ext}, i}(t)\right\}^{T}=\left[1 \frac{m_{2}}{m_{1}} \frac{m_{3}}{m_{1}} \frac{m_{4}}{m_{1}} \frac{m_{5}}{m_{1}}\right] \cdot F_{\mathrm{ext}, 1}(t) .
$$

The considered external forces are the first two types of forces given by Table 2 of the example of Section 3.1.

The mechanical system in [22] is shown to form a perpetual mechanical system, and the solution in the exact augmented perpetual manifold is given by equation (6) with $m=m_{1} / \sigma_{i}$.

The nonlinear system of equations (38) has been solved numerically. All numerical simulations in this section have been performed, with 64-bit Scilab 6.6.1 [27] using "Adams" solver with time step $5 \cdot 10^{-4} \mathrm{~s}$, as well as relative and absolute tolerance $6 \cdot 10^{-14}$.

The system parameters, which define the matrices in equation (35), are shown in Table 6.

The external forces parameters are the same as those given in Table 3 for the first two time intervals of the 2-DOF mechanical system in the previous example. These forces lead to the same characteristic differential equation, the first external force is multiplied with a constant $\sigma_{1}=200$, and the second external force with a constant $\sigma_{2}=400$, which leads to the same vector fields describing the motion for the first two time intervals of the 2-DOF mechanical system example of Section 3.1.

In Figure 9(a), selected numerically determined displacements of the 5-DOF system, of the two edges $\left(x_{1}, x_{5}\right)$ and of the middle $\left(x_{3}\right)$, are depicted incorporating the analytical solution $\left(x_{a}\right)$ and the numerical solution $(x-$ 2dof) of the 2-DOF system of the example of Section 3.1, and it seems that they are in good agreement. This is certified by examining (a) the maximum of the maxima of the absolute differences between the analytical solution and each of the displacements of the 5-DOF system, which has the minimal value of $4.66910^{-8} \mathrm{~m}$, and (b) the maximum of the maxima of the absolute differences between the numerically determined 2-DOF displacement and each of the displacements of the 5-DOF system, which has the minimal value of 4.204 $10^{-8} \mathrm{~m}$.

Some selected numerically determined velocities of the 5-DOF system of the two edges $\left(\dot{x}_{1}, \dot{x}_{5}\right)$ and of the middle $\left(\dot{x}_{3}\right)$ are depicted in Figure 9(b), incorporating the analytical solution $\left(\dot{x}_{a}\right)$ and the numerical solution $(\dot{x}-2$ dof $)$ of the 2 -DOF system of the example of Section 3.1, and it seems that they are in good agreement. This is certified by examining firstly the maximum of the maxima of the absolute differences between the analytical velocity and each of the velocities of the 5-DOF system, which has the minimal value of $1.8410^{-7} \mathrm{~m} / \mathrm{s}$. Secondly, the maximum of the maxima of 
TABLE 6: The parameters of the matrices defining the equations of motion of the train.

\begin{tabular}{lccrc}
\hline $\mathbf{M}_{\mathbf{i}, \mathbf{j}}$ & $\mathbf{K}_{\mathbf{i}, \mathbf{j}}$ & $\mathbf{C}_{\mathbf{i}, \mathbf{j}}$ & & $F_{i}^{\mathrm{NL}}$ \\
\hline$m_{1}=2000 \mathrm{~kg}$ & $k_{1}=1 \cdot 10^{5} \mathrm{~N} / \mathrm{m}$ & $c_{1}=319.07 \mathrm{~N} \cdot \mathrm{s} / \mathrm{m}$ & $k_{n l, 1}=1 \cdot 10^{4} \mathrm{~N}$ & $c_{n l, 1}=319.07 \mathrm{~N} \cdot \mathrm{s}^{3} / \mathrm{m}^{3}$ \\
$m_{2}=1000 \mathrm{~kg}$ & $k_{2}=1.4 \cdot 10^{5} \mathrm{~N} / \mathrm{m}$ & $c_{2}=446.70 \mathrm{~N} \cdot \mathrm{s} / \mathrm{m}$ & $k_{n l, 2}=-1.5 \cdot 10^{4} \mathrm{~N} / \mathrm{m}^{5}$ & $c_{n l, 2}=446.70 \mathrm{~N} \cdot \mathrm{s}^{5} / \mathrm{m}^{5}$ \\
$m_{3}=1500 \mathrm{~kg}$ & $k_{3}=1.3 \cdot 10^{5} \mathrm{~N} / \mathrm{m}$ & $c_{3}=414.80 \mathrm{~N} \cdot \mathrm{s} / \mathrm{m}$ & $k_{n l, 3}=1.3 \cdot 10^{4} \mathrm{~N}$ & $c_{n l, 3}=414.80 \mathrm{~N} \cdot \mathrm{s}^{7} / \mathrm{m}^{7}$ \\
$m_{4}=1200 \mathrm{~kg}$ & $k_{4}=1.2 \cdot 10^{5} \mathrm{~N} / \mathrm{m}$ & $c_{4}=382.88 \mathrm{~N} \cdot \mathrm{s} / \mathrm{m}$ & $k_{n l, 4}=1.2 \cdot 10^{4} \mathrm{~N} / \mathrm{m}^{7}$ & $c_{n l, 4}=382.88 \mathrm{~N}$ \\
$m_{5}=500 \mathrm{~kg}$ & - & - & - & - \\
\hline
\end{tabular}

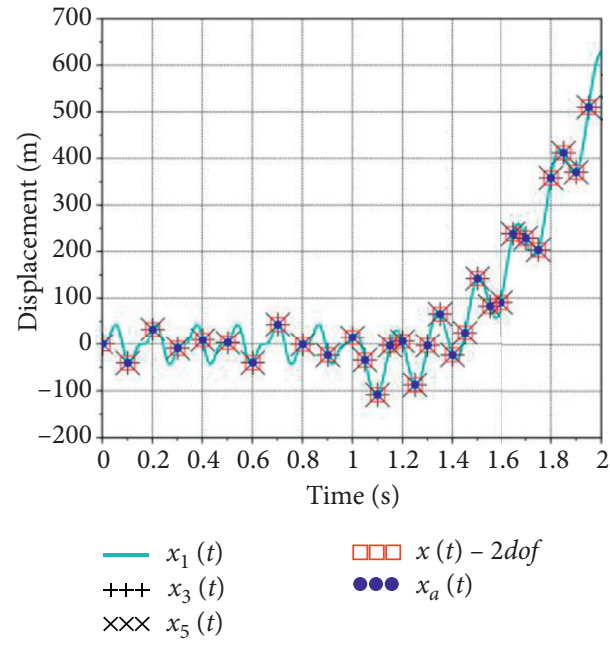

(a)

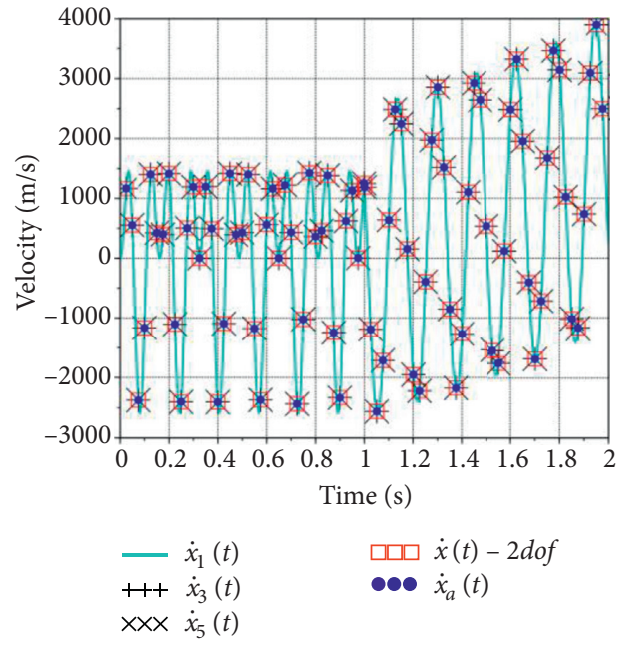

(b)

FIGURE 9: (a) Selected numerically determined displacements of the 5-DOF mechanical system, of the two edges $\left(x_{1}, x_{3}\right)$ and of the middle $\left(x_{5}\right)$, incorporating the analytical solution $\left(x_{a}\right)$ and the 2-DOF mechanical system numerical solution $(x-2$ dof). (b) Selected numerically determined displacements of the 5-DOF mechanical system, of the two edges $\left(\dot{x}_{1}, \dot{x}_{3}\right)$ and of the middle $\left(\dot{x}_{5}\right)$, incorporating the analytical solution $\left(\dot{x}_{a}\right)$ and the 2-DOF mechanical system's numerical solution $(\dot{x}-2$ dof $)$.

the absolute differences between the numerically determined 2 -DOF velocity and each of the velocities of the 5-DOF system, which has the minimal value of $1.9510^{-7} \mathrm{~m} / \mathrm{s}$.

Therefore, the corollary is certified.

\section{Conclusions}

As a continuation of the main theorem defining the conditions for a mechanical system's motion to belong in the exact augmented perpetual manifolds, some theoretical developments with the definition of a corollary and its proof are presented. More precisely, all the perpetual mechanical systems with the same characteristic differential equation have the same general solution. Moreover, the analytical solutions in the exact augmented perpetual manifolds and their augmented perpetual submanifolds for six types of forces are determined. The analytical results are examined with numerical simulations in three examples, and they are in very good agreement.

The significance of this outcome is that the many different mechanical systems, in the exact augmented perpetual manifolds with the same characteristic differential equation, have the same general solution, independent of the type of system, and upon the external forcing can lead to particle- single-frequency or multiple frequencies' standing wave or motion directed single-frequency or multiple frequencies' wave motions.

This work is significant in mathematics for unifying specific solutions of nonautonomous systems of differential equations. Also, in physics/mechanics, the particlewave motions are of high significance. In mechanical engineering, the rigid body motions without any vibrations are the ultimate motions for some flexible mechanical systems.

Further work can be done incorporating the Part-II article of the augmented perpetual manifolds outcome, which is associated with the energies of the mechanical systems in the exact augmented perpetual manifolds.

\section{Appendix}

\section{A. Structural Matrices for Finite Element Formulation}

In this appendix, the structural matrices for the finite element model of the rod with NES attachment are defined.

The mass matrix is given by 


$$
\left[I_{s}\right]=\frac{I \cdot l_{e}}{6} \cdot\left[\begin{array}{ccccccc}
2 & 1 & 0 & 0 & 0 & 0 & 0 \\
1 & 4 & 1 & 0 & 0 & 0 & 0 \\
0 & 1 & 4 & 1 & 0 & 0 & 0 \\
0 & 0 & 1 & 4 & 1 & \vdots & \vdots \\
0 & 0 & 0 & 1 & \ddots & 1 & 0 \\
0 & 0 & 0 & \ldots & 1 & 2 & 0 \\
0 & 0 & 0 & \ldots & 0 & 0 & \frac{6 \cdot I_{\text {nes }}}{I \cdot l_{e}}
\end{array}\right] \text {, }
$$

where $l_{e}$ is the length element, the polar moment of inertia of the NES is $\left(I_{\text {nes }}\right)$, and the distributed (associated with the partial differential equation describing the torsional dynamics) polar moment of inertia of the shaft is denoted by (I) and correlated with the second moment of area $\left(I_{x x}\right)$ through the following formula:

$$
I=\rho \cdot I_{x x}=\rho \cdot \pi \cdot\left(\frac{D_{o}{ }^{4}-D_{i}{ }^{4}}{64}\right) .
$$

The stiffness matrix is given by

$$
\left[K_{r}\right]=\frac{G \cdot I_{x x}}{l_{e}} \cdot\left[\begin{array}{ccccccc}
1 & -1 & 0 & 0 & 0 & 0 & 0 \\
-1 & 2 & -1 & 0 & 0 & 0 & 0 \\
0 & -1 & 2 & -1 & 0 & 0 & 0 \\
0 & 0 & -1 & 2 & -1 & \vdots & \vdots \\
0 & 0 & 0 & -1 & \ddots & -1 & 0 \\
0 & 0 & 0 & \ldots & -1 & 1+\frac{k_{l, \mathrm{nes}} \cdot l_{e}}{G \cdot I_{x x}} & -\frac{k_{l, \mathrm{nes}} \cdot l_{e}}{G \cdot I_{x x}} \\
& & & & & & \\
0 & 0 & 0 & \ldots & 0 & -\frac{k_{l, \mathrm{nes}} \cdot l_{e}}{G \cdot I_{x x}} & \frac{k_{l, \mathrm{nes}} \cdot l_{e}}{G \cdot I_{x x}}
\end{array}\right] \text {. }
$$

The damping matrix is obtained as Rayleigh damping:

$$
\left[C_{r}\right]=\alpha \cdot\left[M_{r}\right]+\beta_{1} \cdot\left[K_{r}\right]
$$

with $\alpha=0$.

\section{Data Availability}

The data can be provided upon request to the author.

\section{Conflicts of Interest}

The author declares that there are no conflicts of interest.

\section{References}

[1] A. Prasad, "Existence of perpetual points in nonlinear dynamical systems and its applications," International Journal of Bifurcation and Chaos, vol. 25, no. 2, Article ID 1530005, 2015.

[2] P. Brzeski and L. N. Virgin, "Experimental Investigation of perpetual points in mechanical systems," Nonlinear Dynamics, vol. 90, no. 4, pp. 2917-2928, 2017.

[3] D. Dudkowski, A. Prasad, and T. Kapitaniak, "Perpetual points and periodic perpetual loci in maps," Chaos: An Interdisciplinary Journal of Nonlinear Science, vol. 26, no. 10, Article ID 103103, 2016.

[4] A. Prasad, "A note on topological conjugacy for perpetual points," International Journal of Applied Nonlinear Science, vol. 21, no. 1, pp. 60-64, 2016.

[5] D. Dudkowski, A. Prasad, and T. Kapitaniak, "Perpetual points and hidden attractors in dynamical systems," Physics Letters A, vol. 379, no. 40-41, pp. 2591-2596, 2015.

[6] D. Dudkowski, S. Jafari, T. Kapitaniak, N. V. Kuznetsov, G. A. Leonov, and A. Prasad, "Hidden attractors in dynamical systems," Physics Reports, vol. 637, pp. 1-50, 2016.

[7] D. Dudkowski, A. Prasad, and T. Kapitaniak, "Perpetual points: new tool for localization of coexisting attractors in dynamical systems," International Journal of Bifurcation and Chaos, vol. 27, no. 4, Article ID 1750063, 2017.

[8] F. Nazaimehr, B. Saaeidi, S. Jafari, and J. C. Sprott, "Are perpetual points sufficient for locating hidden attractors?" International Journal of Bifurcation and Chaos, vol. 27, no. 3, Article ID 1750037, 2017.

[9] F. Nazaimehr, S. Jafari, S. M. R. H. Golpayegani, and J. C. Sprott, "Categorizing chaotic flows from the viewpoint of fixed points and perpetual points," International Journal of Bifurcation and Chaos, vol. 27, no. 2, Article ID 1750023, 2017.

[10] D. Dudkowski, A. Prasad, and T. Kapitaniak, "Describing chaotic attractors: regular and perpetual points," Chaos: An Interdisciplinary Journal of Nonlinear Science, vol. 28, no. 3, Article ID 033604, 2018.

[11] N. V. Kuznetsov, G. A. Leonov, T. N. Mokaev, A. Prasad, and M. D. Shrimali, "Finite-time Lyapunov dimension and hidden attractor of the Rabinovich system," Nonlinear Dynamics, vol. 92, no. 2, pp. 267-285, 2018.

[12] S. N. Chowdhurry and D. Ghosh, "Hidden attractors: a new chaotic system without equilibria," The European Physical Journal Special Topics, vol. 229, pp. 1299-1308, 2020.

[13] F. Yu, Z. Zhang, L. Liu et al., "Secure communication scheme based on a new 5D multistable four-wing memristive hyperchaotic system with disturbance inputs," Complexity, vol. 2020, Article ID 5859273, 2020.

[14] S. Jafari, F. Nazarimehr, J. C. Sprott, and S. M. R. H. Golpayegani, "Limitation of perpetual points for confirming conservation in dynamical systems," International Journal of Bifurcation and Chaos, vol. 25, no. 13, Article ID 1550182, 2015.

[15] S. Cang, A. Wu, Z. Wang, and Z. Chen, "Four-dimensional autonomous dynamical systems with conservative flows: twocase study," Nonlinear Dynamics, vol. 89, no. 4, pp. 2495-2508, 2017.

[16] P. Brzeski and L. N. Virgin, "System identification of energy dissipation in a mechanical model undergoing high velocities: an indirect use of perpetual points," Mechanical Systems and Signal Processing, vol. 108, pp. 115-125, 2018.

[17] A. Wu, S. Cang, R. Zhang, Z. Wang, and Z. Chen, "Hyperchaos in a conservative system with nonhyperbolic fixed points," Complexity, vol. 2018, Article ID 9430637, 2018. 
[18] N. Wang, G. Zhang, and H. Bao, "Infinitely many coexisting conservative flows in a $4 \mathrm{D}$ conservative system inspired by LC circuit," Nonlinear Dynamics, vol. 99, no. 4, pp. 3197-3216, 2020.

[19] F. Georgiades, "Theorem and observation about the nature of perpetual points in conservative mechanical systems," IUTAM Symposium on Exploiting Nonlinear Dynamics for Engineering Systems, vol. 37, pp. 91-104, 2020.

[20] F. Georgiades, "Perpetual points in natural mechanical systems with viscous damping: a theorem and a remark," Proceedings of the Institution of Mechanical Engineers, Part C: Journal of Mechanical Engineering Science, 2020.

[21] F. Georgiades, "Augmented perpetual manifolds, a corollary: dynamics of natural mechanical systems with eliminated internal forces," in Proceedings of NODYCON 21, NODYCON, Rome, Italy, February 2021.

[22] F. Georgiades, “Augmented perpetual manifolds and perpetual mechanical systems-Part I: definitions, theorem and corollary for triggering perpetual manifolds, application in reduced order modeling and particle-wave motion of flexible mechanical systems," ASME Journal of Computational and Nonlinear Dynamics, vol. 16, no. 7, p. 19, 2021.

[23] J. Kaplunov, A. Shestakova, I. Aleynikov, B. Hopkins, and A. Talonov, "Low-frequency perturbations of rigid body motions of a viscoelastic inhomogeneous bar," Mechanics of Time-Dependent Materials, vol. 19, no. 2, pp. 135-151, 2015.

[24] J. Kaplunov and O. Şahin, "Perturbed rigid body motions of an elastic rectangle," Zeitschrift für angewandte Mathematik und Physik, vol. 71, no. 5, pp. 1-15, 2020.

[25] R. I. Leine and H. Nijmeier, Dynamics and Bifurcations of Non-Smooth Mechanical Systems, Springer, Berlin, Heidelberg, Germany, 2004.

[26] A. F. Filippov, Differential Equations with Discontinuous Righthand Sides, Springer Science+Business Media, Dordrecht, Netherlands, 1988.

[27] Scilab: Version 5.5.2, The Scilab Team, 2015, https://scilab.org.

[28] F. Georgiades, Nonlinear Localization and Targeted Energy Transfer Phenomena in Vibrating Systems with Smooth and Non-Smooth Stiffness Nonlinearities, Ph.D. thesis, National Technical University of Athens, Greece, 2006.

[29] M. Geradin and D. Rixen, Mechanical Vibrations, John Wiley \& Sons, Hoboken, NJ, USA, 1997. 